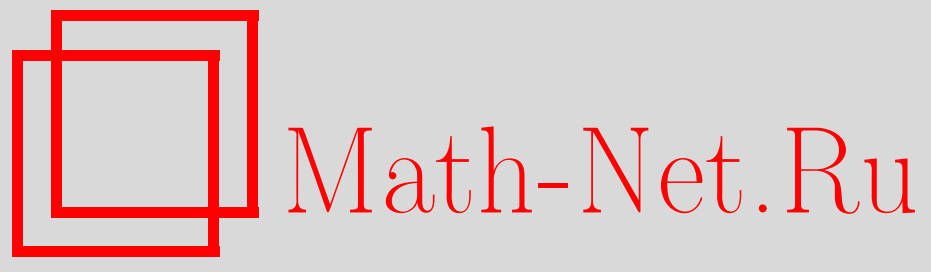

Р. Н. Карасёв, Топологические методы в комбинаторной геометрии, УМH, 2008, том 63, выпуск 6, 39-90

DOI: https://doi.org/10.4213/rm9244

Использование Общероссийского математического портала Math-Net.Ru подразумевает, что вы прочитали и согласны с пользовательским соглашением http://www . mathnet.ru/rus/agreement

Параметры загрузки:

IP: 54.162 .27 .143

26 апреля 2023 г., 13:43:20

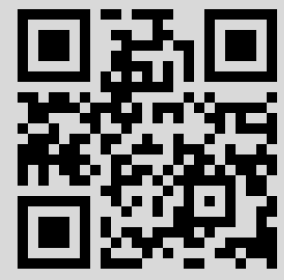




\section{Топологические методы в комбинаторной геометрии}

\section{P. H. Kapacëв}

Обзор посвящен некоторым результатам в области комбинаторной и выпуклой геометрии, начиная с классических теорем и вплоть до последних современных результатов. В основном рассматриваются те результаты, в доказательстве которых существенно применяются методы алгебраической топологии.

Подробно освещаются разные обобщения теоремы Борсука-Улама для $\left(Z_{p}\right)^{k}$-действия, применения к задаче Кнастера об уровнях функции на сфере, обсуждаются приложения к теории Люстерника-Шнирельмана оценки количества критических точек гладкой функции.

Дается обзор топологических методов в оценках хроматического числа графов и гиперграфов, в теоремах типа Тверберга и ван КампенаФлореса. Приводятся описания результатов автора по "двойственным" аналогам теорем о центральной точке и Тверберга.

Рассматриваются результаты о существовании вписанных и описанных многогранников специального вида для выпуклых тел, о существовании бильярдных траекторий в выпуклом теле. Приводятся результаты о делении мер гиперплоскостями и другими разбиениями евклидова пространства.

Дается краткий обзор топологических подходов к теоремам типа Хелли, связанных с рассмотрением нерва семейств выпуклых множеств в евклидовом пространстве.

Приводится обзор по теоремам типа Хелли для плоских трансверсалей, подробно рассматриваются результаты, использующие топологию многообразия Грассмана и канонического расслоения над ним.

Библиография: 137 названий.

СОДЕРЖАНИЕ

1. Введение......................................... 40

2. Классические теоремы комбинаторной и выпуклой геометрии ....... 41

2.1. Теорема Брауэра о неподвижной точке ................. 41

2.2. Теоремы Каратеодори, Радона и Хелли................... 41

2.3. Теорема Кнастера-Куратовского-Мазуркевича и лемма Шпернера о триангуляции симплекса .................. 42

(C) P. H. KAPACËB, 2008 
2.4. Теоремы Борсука-Улама, Люстерника-Шнирельмана, теорема "о бутерброде" .............................. 43

2.5. Теорема Неймана-Радо о центральной точке ............... 44

2.6. Теоремы Шнирельмана и Какутани о вписывании и описывании 44

3. Топологические обобщения теоремы Борсука-Улама .............. 44

3.1. Пространства с действием конечной группы............... 44

3.2. Теорема ван Кампена-Флореса ....................... 45

3.3. Теоремы типа Борсука-Улама для действия групп $\left(Z_{p}\right)^{k} \ldots \ldots .45$

3.4. Теоремы типа Борсука-Улама для других конечных групп ..... 46

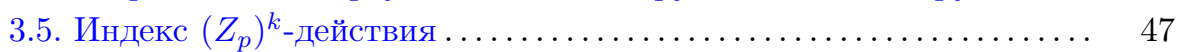

3.6. $\left(Z_{p}\right)^{k}$-действие в задаче Кнастера................... 50

3.7. Теория Люстерника-Шнирельмана для функций с симметриями 51

4. Топологические методы и обобщения классических теорем комбинатор-

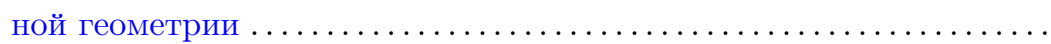

4.1. Топологические методы оценки хроматического числа графов и гиперграфов ..................................... 52

4.2. Теорема Тверберга, “цветные” теоремы Каратеодори и Хелли . . 55

4.3. Интерпретация теорем существования в терминах препятствий и относительный класс Эйлера ................. 56

4.4. Топологические теоремы типа Тверберга, обобщения теоремы ван Кампена - Флореса ........................... 59

4.5. Двойственные теоремы о центральной точке и Тверберга ...... 61

4.6. Теоремы существования для вписанных и описанных фигур .... 62

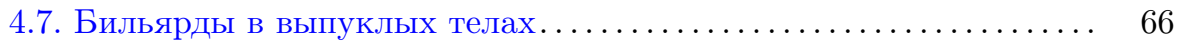

4.8. Деление мер на равные части и части заданной меры ........ 67

4.9. Топологический подход к теоремам типа Хелли ............. 72

4.10. Теоремы типа Кнастера-Куратовского-Мазуркевича ........ 75

5. Плоские трансверсали и топология многообразий Грассмана........ 77

5.1. Теоремы типа Хелли для плоских трансверсалей............ 77

5.2. Топология канонического расслоения над многообразием Грас-

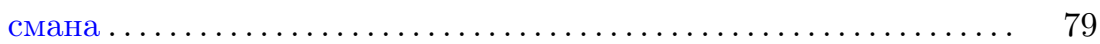

5.3. Теорема Дольникова и другие теоремы типа Хелли о плоских трансверсалях ................................ 80

5.4. Теорема об общей неподвижной точке послойных отображений . 82

5.5. Теоремы о центральной точке и Тверберга для трансверсалей .. 82

5.6. Теоремы типа Борсука-Улама для трансверсалей ........... 83

Список литературы . . . . . . . . . . . . . . . . . . 84

\section{1. Введение}

В этом обзоре рассматриваются разные варианты применения топологических методов в задачах комбинаторной и выпуклой геометрии. Внимание к топологическим методам неслучайно, ведь одни из самых первых классических 
теорем комбинаторной и выпуклой геометрии - лемма Шпернера о триангуляции симплекса, теорема Хелли, теорема "о бутерброде" - имеют непосредственное отношение к топологии.

Следует отметить, что в начале двадцатого века связь между топологией и комбинаторной геометрией выражалась в основном в поисках комбинаторных доказательств основных топологических фактов, связанных с понятием размерности и степени отображения. Типичным примером является уже упомянутая лемма Шпернера. В последнее время наблюдается в основном обратный процесс: содержательные топологические факты находят приложения в комбинаторике и комбинаторной геометрии и часто позволяют по-новому взглянуть на старые результаты.

\section{2. Классические теоремы комбинаторной и выпуклой геометрии}

2.1. Теорема Брауэра о неподвижной точке. Одним из результатов, демонстрирующих важность топологических методов в геометрии, является следующая теорема [1].

Теорема 1 (Брауэр, 1910). Пусть $K \subseteq \mathbb{R}^{d}$ - выпуклый компакт. Тогда для всякого непрерывного отображения $f: K \rightarrow K$ найдется точка $x \in K$ такая, что $x=f(x)$. Такая точка называется неподвижной точкой отображения $f$.

В современной интерпретации, теорема Брауэра следует из существования некоторого препятствия к построению отображения без неподвижной точки, которое по сути является классом Эйлера векторного расслоения. Более подробное обсуждение такого подхода и пример доказательства теоремы Брауэра см. в п. 4.3 .

2.2. Теоремы Каратеодори, Радона и Хелли. Одним из основных фактов о понятии выпуклости является теорема Каратеодори [2].

Теорема 2 (Каратеодори, 1911). Если $X$ - конечное множество в $\mathbb{R}^{d}$, то всякая точка его выпуклой оболочки лежит в выпуклой оболочке некоторого подмножества $Y \subseteq X$, для которого $|Y| \leqslant d+1$.

Здесь и далее $|Y|$ обозначает число элементов множества $Y$.

Другими важными утверждениями про выпуклость являются теоремы Радона и Хелли (см. работы [3], [4]). Теорема Хелли была доказана Хелли (примерно в 1915-1916 г.) и сообщена Радону, Радон первым опубликовал придуманное им доказательство теоремы Хелли через следующую теорему.

Теорема 3 (Радон, 1921). Всякое множество из $d+2$ точек в $\mathbb{R}^{d}$ можно разбить на два непустых подмножества, выпуклые оболочки которых пересекаются.

ТеОрема 4 (Хелли, 1923). Пусть $\mathscr{F}$ - конечное семейство выпуклых множеств в $\mathbb{R}^{d}$. Семейство $\mathscr{F}$ имеет общую точку тогда и только тогда, когда всякое подсемейство $\mathscr{G} \subseteq \mathscr{F}$ с $|\mathscr{G}| \leqslant d+1$ имеет обиую точку. 
Теорема Хелли может быть также выведена из теоремы Каратеодори. Кроме того, теорема Хелли допускает топологическое доказательство, которое будет обсуждено в п. 4.9 .

\section{3. Теорема Кнастера-Куратовского-Мазуркевича и лемма Шпе-} рнера о триангуляции симплекса. Сформулируем лемму Шпернера о триангуляции симплекса [5]. Сначала зафиксируем некоторые обозначения.

ОПРЕДЕЛЕНИЕ 1. Симплексом размерности $n$ назовем выпуклую оболочку $n+1$ аффинно независимой точки $x_{1}, \ldots, x_{n+1}$ в $\mathbb{R}^{d}(d \geqslant n)$.

Для симплекса $\Delta=\operatorname{conv}\left\{x_{1}, \ldots, x_{n+1}\right\}$ выпуклые оболочки

$$
\partial_{i} \Delta=\operatorname{conv}\left\{x_{1}, \ldots, \hat{x}_{i}, \ldots, x_{n+1}\right\}
$$

назовем гипергранями симплекса. Выпуклые оболочки непустых собственных подмножеств множества $\left\{x_{1}, \ldots, x_{n+1}\right\}$ назовем гранями симплекса. Сами точки $x_{1}, \ldots, x_{n+1}$ назовем вершинами симплекса.

ОПРедЕЛЕНиЕ 2. Обозначим множество индексов $[m]=\{1,2, \ldots, m\}$. Pacкраской множества $X$ в $m$ цветов назовем отображение $c: X \rightarrow[m]$. Если $c(x)=i$, то будем говорить, что $x$ покрашено в цвет $i$.

ОПРедЕЛЕНиЕ 3. Триангуляцией подмножества $X \subseteq \mathbb{R}^{d}$ назовем набор симплексов $T$ такой, что

1) если $S \in T$, то все грани $S$ тоже принадлежат $T$;

2) если $S_{1}, S_{2} \in T$, то пересечение $S_{1} \cap S_{2}$ либо пусто, либо является гранью $S_{1}$ и гранью $S_{2}$;

3) $X=\bigcup T$.

Грани симплексов триангуляции называются гранями триангуляции, ребра симплексов триангуляции называются ребрами триангуляции.

Теорема 5 (Шпернер, 1928). Пусть $\Delta$ - симплекс размерности $n$ и $T$ триангуляиия симплекса $\Delta$, вершины которой раскрашены в $n+1$ ивет так, что на гиперграни $\partial_{i} \Delta$ не встречается ивет $i$. Тогда триангуляция $T$ содержит симплекс, на вершинах которого встречаются все иявета.

Непрерывным аналогом леммы Шпернера является теорема Кнастера-Куратовского-Мазуркевича (далее просто теорема KKM) [6].

Теорема 6 (Кнастер, Куратовский, Мазуркевич, 1929). Предположим, что $n$-мерный симплекс $\Delta$ покрыт $n+1$ замкнутым подмножеством $F_{1}, \ldots, F_{n+1}$, причем для каждого $i \in[n+1]$

$$
F_{i} \cap \partial_{i} \Delta=\varnothing
$$

Тогда $\bigcap_{i=1}^{n+1} F_{i} \neq \varnothing$.

Теорема KKM следует из леммы Шпернера, а из теоремы KKM, в свою очередь, следует теорема Брауэра о неподвижной точке. Доказательство теоремы Брауэра о неподвижной точке через лемму Шпернера дает некоторый способ вычислительной реализации теоремы Брауэра.

Приведем также близкую к лемме Шпернера лемму из [7]. 
Теорема 7 (Таккер, 1946). Пусть Т - триангуляция центрально-симметричного выпуклого многогранника $K \subset \mathbb{R}^{d}$, централъно симметричная на $\partial K$. Предположим, что на всех вершинах Т расставлены метки из множества $\{+1,-1,+2,-2, \ldots,+d,-d\}$, причем для всякой вершины $v \in \partial K$ сумма меток $v$ и $-v$ равна нулю. Тогда найдется ребро триангулячии T, сумма меток на концах которого равна нулю.

С помощью этой леммы можно доказать не только теорему Брауэра, но и теорему Борсука-Улама, о которой пойдет речь в следующем пункте.

\section{4. Теоремы Борсука-Улама, Люстерника-Шнирельмана, теоре-} ма “о бутерброде". Приведем три эквивалентных формулировки теоремы Борсука-Улама-Люстерника-Шнирельмана [8], [9], которая тесно связана с теоремами Хелли и Брауэра.

Теорема 8 (Борсук, Люстерник, Шнирельман, 1930-1933). Если сфера $S^{n}$ покрыта $n+1$ замкнутым множеством $X_{1}, \ldots, X_{n+1}$, то по крайней мере одно из $X_{i}$ содержит пару диаметрально противоположных точек на сфере.

Теорема 9 (Борсук, Улам, 1933). Если на сфере $S^{n}$ даны $n$ нечетных непрерывных функций $f_{1}, \ldots, f_{n}$, то для некоторой точки $x \in S^{n}$

$$
f_{1}(x)=\cdots=f_{n}(x)=0 .
$$

ТеОрема 10 (Борсук, Улам, 1933). Для всякого непрерывного отображения $f: S^{n} \rightarrow \mathbb{R}^{n}$ найдется точка $x \in S^{n}$, для которой $f(x)=f(-x)$.

Из теоремы Борсука-Улама также можно вывести теорему Брауэра о неподвижной точке.

Следующее утверждение также эквивалентно теореме Борсука-Улама.

Теорема 11 (Люстерник, Шнирельман, 1930). Если сфера $S^{n}$ nокрыта $n$ замкнутыми множествами $X_{1}, \ldots, X_{n}$, инвариантными относительно иентральной симметрии $x \mapsto-x$, то для некоторого $i$ одна из компонент связности множества $X_{i}$ содержит пару диаметрально противоположных точек на сфере.

В п. 3.7 будет приведено небольшое обсуждение теории Люстерника-Шнирельмана, обобщения и применения теоремы 11.

Одним из геометрических следствий теоремы Борсука-Улама является следующая теорема о делении мер [10], [11].

Теорема 12 (“о бутерброде”; Стоун, Тьюки, Штейнгауз, 1942-1945). Пусть на $\mathbb{R}^{d}$ заданы d абсолютно непрерывных вероятностных мер $\mu_{1}, \ldots, \mu_{d}$. Тогда найдется полупространство $H \subset \mathbb{R}^{d}$ такое, что для любого $i=1, \ldots, d$

$$
\mu_{i}(H)=\frac{1}{2} .
$$

Теорема Борсука-Улама для покрытий допускает другую формулировку: $(d-1)$-мерную сферу диаметра 1 нельзя покрыть $d$ множествами диаметра меньшего 1. На основе этого факта Борсук сформулировал гипотезу. 
ГиПотезА 1 (Борсук, 1933). Любой выпуклый компакт $K \subset \mathbb{R}^{d}$ можно разбить на $d+1$ множество менъшего диаметра.

Эта гипотеза и близкие к ней вопросы фигурируют в литературе под названием "проблема Борсука". Для гладких компактов гипотеза Борсука доказывается довольно просто, но в случае негладких компактов был обнаружен контрпример [12], более того, оказалось, что порядок роста требуемого количества множеств разбиения не менее $1.2^{\sqrt{d}}$, что сильно отличается от предполагаемых $d+1$ при достаточно больших $d$. В п. 4.6 будут обсуждаться варианты доказательства гипотезы Борсука и ее усилений в размерностях 2 и 3.

2.5. Теорема Неймана-Радо о центральной точке. Другой результат, касающийся деления мер гиперплоскостями, - теорема о центральной точке [13], [14].

Теорема 13 (теорема о центральной точке; Нейман, Радо, 1945-1946). Для абсолютно непрерывной вероятностной мерь $\mu$ на $\mathbb{R}^{d}$ найдется такая точка $x \in \mathbb{R}^{d}$, что для всякого полупространства $H \ni x$ выполнено неравенство

$$
\mu(H) \geqslant 1 /(d+1) .
$$

Эта теорема является следствием теоремы Хелли. У теоремы о центральной точке также есть дискретный аналог.

ТЕОРема 14 (дискретная теорема о центральной точке). Пусть в $\mathbb{R}^{d}$ дано конечное множество $X$ из $n$ точек. Тогда найдется точка $x \in \mathbb{R}^{d}$ такая, что для всякой полуплоскости $H \ni x$

$$
|H \cap X| \geqslant\left\lfloor\frac{n+d}{d+1}\right\rfloor .
$$

2.6. Теоремы Шнирельмана и Какутани о вписывании и описывании. Приведем два классических результата из работ [15], [16] о вписывании и описывании фигур, доказательства которых основываются на топологических соображениях.

Теорема 15 (Шнирельман, 1934). Для всякой гладкой замкнутой кривой $C \subset \mathbb{R}^{2}$ без самопересечений найдется квадрат, все вериины которого лежат на кривой $C$.

Теорема 16 (Какутани, 1942). Для всякого компакта $K \subset \mathbb{R}^{3}$ найдется куб $Q$, содержащии $K$, каждая гранъ которого пересекается с $K$.

В п. 4.6 приведены другие теоремы о вписывании и описывании, а также приведено доказательство теоремы Шнирельмана с помощью современного топологического подхода.

\section{3. Топологические обобщения теоремы Борсука-Улама}

3.1. Пространства с действием конечной группы. Сначала введем некоторую терминологию, которая позволит дать еще одну формулировку теоремы Борсука-Улама и ее обобщений. Далее $G$ - некоторая конечная группа. 
ОПРЕДЕЛЕНИЕ 4. Топологическое пространство с непрерывным действием группы $G$ называется $G$-пространством. Непрерывные отображения между $G$-пространствами, коммутирующие с действием $G$, назовем $G$-отображениями или $G$-эквивариантными отображениями.

Далее циклические группы порядка $n$ обозначаем $Z_{n}$. В этих терминах теорема Борсука-Улама формулируется следующим образом. Пусть группа $Z_{2}$ действует на $n$-мерных сферах отображением $x \mapsto-x$, далее такое действие будем называть антиподальным.

ТеОрема 17 (теорема Борсука-Улама для $Z_{2}$ ). He существует $Z_{2}$-отображений сфер с антиподальным действием $S^{n} \rightarrow S^{k}$, если $n>k$.

3.2. Теорема ван Кампена-Флореса. Следствием теоремы БорсукаУлама в приведенной выше формулировке является следующая теорема о невложимости [17], [18].

Теорема 18 (ван Кампен, Флорес, 1932-1934). Пусть $\Delta_{k}^{2 k+2}-k$-мерный остов $(2 k+2)$-мерного симплекса. Тогда не существует непрерывного ингективного отображения

$$
f: \Delta_{k}^{2 k+2} \rightarrow \mathbb{R}^{2 k}
$$

ДокАЗАТЕЛЬСтво. Обозначим $K=\Delta_{k}^{2 k+2}$. Инъективное отображение $f$ : $K \rightarrow \mathbb{R}^{2 k}$ порождает отображение

$$
g(x, y) \mapsto x-y,
$$

которое после нормализации можно считать отображением из множества пар точек $(x, y) \in K \times K$ с непересекающимися носителями в сферу $S^{2 k-1}$. Это отображение коммутирует с действием группы $Z_{2}$ перестановками на $K \times K$ и антиподальным действием на $S^{2 k-1}$. При этом можно показать, что пространство пар точек с непересекающимися носителями (вырезанное произведение, см. п. 4.4) $K_{\Delta}^{2}$ гомеоморфно $2 k$-мерной сфере с антиподальным действием $Z_{2}$. Значит, отображение $g$ не существует по теореме Борсука-Улама.

3.3. Теоремы типа Борсука-Улама для действия групп $\left(Z_{p}\right)^{k}$. Для распространения теоремы Борсука-Улама от $Z_{2}$-отображений до $G$-отображений с другой конечной группой $G$ следует понять, какими пространствами можно заменить сферы $S^{n}$ и $S^{k}$ и какое действие группы $G$ на них потребовать.

ОПредЕЛЕНиЕ 5 . Пусть $X-G$-пространство. Точка $x \in X$ называется неподвижной точкой группы $G$, если для любого $g \in G$

$$
g x=x .
$$

Множество неподвижных точек $G$-пространства $X$ обозначается $X^{G}$.

Теорема Борсука-Улама для отображений неоднократно обобщалась (см. [19]-[23]). Одна из последних общих формулировок обобщения теоремы Борсука-Улама выглядит следующим образом [24]. Далее $p$ обозначает некоторое простое число. 
Теорема 19 (Воловиков, 1996). Рассмотрим групnу $G=\left(Z_{p}\right)^{k}$ u $G$-nространства $X$ и $Y$. Предположим, что $\widetilde{H}^{i}\left(X, Z_{p}\right)=0$ при всех $i \leqslant n-1, Y^{G}=\varnothing u$

$$
\widetilde{H}^{i}\left(Y, Z_{p}\right)= \begin{cases}0, & i \neq k, \\ Z_{p}, & i=k .\end{cases}
$$

Eсли $n>k$, то не существует $G$-отображений $f: X \rightarrow Y$.

На самом деле в приложениях, как правило, $Y$ - это пространство сфер некоторого линейного представления $V$ группы $G$, которое не имеет тривиальных слагаемых $\left(V^{G}=0\right)$. Доказательство теоремы 19 существенно опирается на тот факт, что класс Эйлера представления $V$ в эквивариантных когомологиях одноточечного пространства $H_{G}^{\operatorname{dim}} V\left(\mathrm{pt}, Z_{p}\right)$ не равен нулю (см. [25; гл. IV, §1]).

Возможно также обобщение теоремы Борсука-Улама в формулировке для отображений $S^{n} \rightarrow \mathbb{R}^{n}$ (ниже приведен частный случай утверждения из [26]).

Теорема 20 (Воловиков, 1992). Рассмотрим группу $G=\left(Z_{p}\right)^{k}$ u $G$-nространство $X$. Пусть $M$ - компактное топологическое ориентируемое, если $p>2$. Рассмотрим непрерывное отображение $f: X \rightarrow M$. Предположим, что $\widetilde{H}^{i}\left(X, Z_{p}\right)=0$ при всех $i \leqslant n-1, n \geqslant m\left(p^{k}-1\right)$, и если $p^{k}=2$, то гомоморфизм $f^{*}: H^{m}\left(M, Z_{p}\right) \rightarrow H^{m}\left(X, Z_{p}\right)$ тривиален. Тогда отображение $f$ отображает некоторую орбиту $G$ в одну точку.

Можно заметить, что в этом утверждении нельзя заменить топологическое многообразие размерности $m$ на симплициальный или $C W$-комплекс размерности $m$ (см. [27], [28]). Простейший пример дает рассмотрение окружности $S$ и триода $T$ - множества, состоящего из трех отрезков с общей точкой. Тогда легко строится непрерывное отображение $S \rightarrow T$, которое не отображает никакие две антиподальные точки $S$ в одну точку $T$.

3.4. Теоремы типа Борсука-Улама для других конечных групп. В книге [29] рассматривался вопрос обобщения теоремы Борсука-Улама на $G$-действия на сферах представлений $G$ с некоторым ослаблением условия на размерности.

ОПРЕДЕЛЕНИЕ 6. Будем говорить, что для группы $G$ выполняется свойство Борсука-Улама с константой $a_{G}$, если для двух линейных представлений $V$ и $W$ группы $G$ с условием $W^{G}=0$ из существования $G$-отображения между пространствами сфер $S V \rightarrow S W$ следует, что $\operatorname{dim} V \leqslant a_{G} \operatorname{dim} W$.

Основной результат можно сформулировать следующим образом [29].

ТеОРема 21 (Барч, 1993). Из конечных групп только р-группъ обладают свойством Борсука-Улама с некоторой константой.

Утверждение для $p$-групп следует из положительного решения [30] гипотезы Сигала об изоморфизме между группой стабильных когомотопий классифицирующего пространства $B G$ и пополнением кольца Бернсайда группы $G$. За подробностями читатель может обратиться к первоисточникам [30], [29], в данном обзоре далее этот факт использоваться не будет. 
В следующем пункте рассматривается более простая и удобная в приложениях топологическая техника, связанная с использованием обычных эквивариантных (борелевских) когомологий.

3.5. Индекс $\left(Z_{p}\right)^{k}$-действия. В приведенных выше формулировках теорем 19 и 20 требовались некоторые условия на $Z_{p}$-связность пространств с действием группы $G=\left(Z_{p}\right)^{k}$.

В некоторых применениях этого условия достаточно и даже удается доказать, что соответствующее пространство $(n-1)$-связно с любыми коэффициентами. Но для ряда применений этого недостаточно. Поэтому требуется более точное описание препятствия к построению $G$-отображения.

Здесь мы приведем краткий обзор, более полное изложение можно найти в книге [25]. Напомним некоторые общепринятые обозначения.

ОПРЕДЕЛЕНИЕ 7. Пусть $E G$ - гомотопически тривиальный $C W$-комплекс с действием группы $G$. В качестве $E G$ для конечной группы можно взять бесконечный (прямой предел конечных) джойн $G * G * \cdots * G * \cdots$. Будем также обозначать $k$-кратный джойн $E G_{k}=G^{* k}$.

Фактор $E G / G$ будем обозначать $B G$.

ОПредЕЛЕНиЕ 8. Для $G$-пространства $X$ обозначим $X_{G}=(X \times E G) / G$, где группа $G$ диагонально действует на произведении (конструкция Бореля). Тогда эквивариантными когомологиями $X$ с коэффициентами в $A$ называются группы

$$
H_{G}^{*}(X, A)=H^{*}\left(X_{G}, A\right) .
$$

Если действие группы $G$ на $X$ свободно, то, очевидно, $X_{G}$ гомотопически эквивалентно $X / G$ и тогда $H_{G}^{*}(X, A)=H^{*}(X / G, A)$. Когомологии в определении могут быть любыми, однако при рассмотрении "сложных" множеств в геометрических задачах бывает полезно использовать когомологии АлександераСпеньера или Чеха, так как они обладают свойством непрерывности относительно пересечений.

Из определения ясно, что эквивариантные когомологии являются контравариантным функтором $G$-пространств. Каждое $G$-пространство $X$ допускает отображение в одноточечное пространство pt с тривиальным действием $G$, следовательно, существует каноническое отображение $\pi_{X}^{*}: H_{G}^{*}(\mathrm{pt}, A)=$ $H^{*}(B G, A) \rightarrow H_{G}^{*}(X, A)$.

Далее рассматриваем случай групп вида $\left(Z_{p}\right)^{k}$ и за поле коэффициентов когомологий берем $Z_{p}$. Обозначение коэффициентов когомологий опускаем. Тогда всякая алгебра когомологий $H_{G}^{*}(X)$ с помощью отображения $\pi_{X}^{*}$ получает естественную структуру алгебры над $A_{G}=H_{G}^{*}(\mathrm{pt})$. Далее будет применяться следующее описание строения алгебры $A_{G}$.

Теорема 22. При $p=2$ алгебра $A_{G}$ является кольцом многочленов от $k$ одномерных образующих $\left\{v_{i}\right\}_{i=1}^{k}$.

При р > 2 алгебра $A_{G}$ является тензорным произведением кольца многочленов от $k$ двумерных образующих $\left\{u_{i}\right\}_{i=1}^{k}$ и внешней алгебры от $k$ одномерных 
образующих $\left\{v_{i}\right\}_{i=1}^{k}$. При этом действие гомоморфизма Бокштейна задается формулой $\beta v_{i}=u_{i}$.

Сделаем определение, следуя работе [31].

ОПРеДЕЛЕНИЕ 9. Для $G$-пространства $X$ ядро естественного отображения $\pi_{X}^{*}: A_{G} \rightarrow H_{G}^{*}(X)$ назовем индексом $X$ со значением в идеалах и обозначим $\operatorname{Ind}_{G} X$.

Иногда бывает удобнее ввести численный индекс.

ОПРедЕЛЕНИЕ 10. Обозначим $I_{n} A_{G}$ градуированный идеал в $A_{G}$, состоящий из элементов размерности $\geqslant n$.

ОПРЕДЕЛЕНИЕ 11. (Нижним) когомологическим индексом $G$-пространства $X$ назовем

$$
\text { ind }_{G} X=\max \left\{n: \operatorname{Ind}_{G} X \subseteq I_{n+1} A_{G}\right\} .
$$

ОПРЕДЕЛЕНИЕ 12. Верхним когомологическим индексом $G$-пространства $X$ назовем

$$
\overline{\operatorname{ind}}_{G} X=\max \left\{n: \exists \alpha \in A_{G}, \pi_{X}^{*}(\alpha) \neq 0\right\} .
$$

Исходя из строения алгебры $A_{G}$ и действия в ней гомоморфизма Бокштейна легко видеть, что в случае группы $G=Z_{p}$ индекс со значением в идеалах полностью определяется численным индексом и оба численных индекса равны. Важность индекса иллюстрируется следующей теоремой.

ТЕОРема 23 (монотонность индекса). Если существует G-отображение $X \rightarrow Y, m o \operatorname{Ind}_{G} X \supseteq \operatorname{Ind}_{G} Y$.

Это утверждение очевидно следует из функториальности естественного отображения $A_{G} \rightarrow H_{G}^{*}(X)$. Оно дает способ доказать отсутствие $G$-отображений между пространствами.

Опишем один из основных способов практического вычисления эквивариантных когомологий и, в частности, индекса (см. также [32]).

Теорема 24 (спектральная последовательность Лере-Серра). Для всякого связного $G$-пространства существует спектральная последовательность с членом

$$
E_{2}^{x, y}=H^{x}\left(B G, \mathscr{H}^{y}\left(X, Z_{p}\right)\right)
$$

сходящаяся к градуированному модулю, соответствующему некоторой фильтрации $H_{G}^{*}\left(X, Z_{p}\right)$. Здесь система коэффичиентов $\mathscr{H}^{y}\left(X, Z_{p}\right)$ получается из когомологий $H^{y}\left(X, Z_{p}\right)$ действием на них $G=\pi_{1}(B G)$. Дифференциалы этой спектральной последовательности являются гомоморфизмами $H^{*}\left(B G, Z_{p}\right)$ модулей.

Это утверждение показывает, что $\operatorname{Ind}_{G} X$ является образом всех дифференциалов спектральной последовательности в нижней строке этой последовательности. В частности, если $\widetilde{H}^{i}\left(X, Z_{p}\right)=0$ при $i \leqslant n-1$, то $\underline{\operatorname{ind}}_{G} X \geqslant n$. Приведем некоторое обобщение этого рассуждения, более слабый вариант которого был использован в работе автора [33]. 
Теорема 25 (Карасёв, 2008). Пусть $G=\left(Z_{p}\right)^{k}, G$-пространство $X$ связно и группь $\widetilde{H}^{m}\left(X, Z_{p}\right)$ при $m<n$ как $Z_{p}[G]$-модули имеют композичионные ряды, составленные из конечномерных $Z_{p}[G]$-модулей, индуцированных с собственных подгрупп $H \subset G$. Тогда

$$
\underline{\text { ind }}_{G} X \geqslant n \text {. }
$$

Напомним, что $Z_{p}[G]$-модуль $M$ индуцирован из $Z_{p}[H]$-модуля $N$, если $M=$ $Z_{p}[G] \otimes_{Z_{p}[H]} N$. Конечномерный модуль индуцирован тогда и только тогда, когда он коиндуцирован:

$$
M=\operatorname{Hom}_{Z_{p}[H]}\left(Z_{p}[G], N\right)
$$

(см. [34; гл. III, предложение 5.9]).

ДокАЗАТЕЛЬСтво. Пусть если $p=2$, то $S_{G}=A_{G}$, а если $p>2$, то $S_{G}=$ $Z_{p}\left[u_{1}, \ldots, u_{k}\right]$, где $Z_{p}\left[u_{1}, \ldots, u_{k}\right]$ - это алгебра многочленов, содержащаяся в $A_{G}$.

Если $G$-модуль $M$ коиндуцирован из $H$-модуля $N$, то $H_{G}^{*}(M)=H_{H}^{*}(N)$ (см. [34; гл. III, предложение 6.2]). Следовательно, для таких модулей многочлен Гильберта их $A_{G}$-модулей когомологий имеет степень не более $k-1$. Если $G$-модуль имеет композиционный ряд из таких модулей, то многочлен Гильберта его когомологий также будет иметь степень не более $k-1$, что следует из рассмотрения длинной точной последовательности когомологий.

Следовательно, строчки с номерами $m=1, \ldots, n-1$ в члене $E_{2}$ спектральной последовательности теоремы 24 имеют многочлены Гильберта степеней не более $k-1$. Это будет верно и во всех остальных членах спектральной последовательности $E_{r}(r \geqslant 2)$. Покажем, что образ всякого дифференциала $d_{2}, \ldots, d_{n-1}$ в нижней строке спектральной последовательности равен нулю. Это следует из того, что если многочлен Гильберта $A_{G}$-модуля $L$ имеет степень не более $k-1$, то

$$
\operatorname{Hom}_{A_{G}}\left(L, A_{G}\right)=0
$$

так как всякий $l \in L$ аннулируется некоторым элементом из алгебры $S_{G}$ (иначе противоречие со степенью многочлена Гильберта), а в модуле $A_{G}$ никакой элемент модуля не аннулируется никаким элементом $S_{G}$.

Значит, в нулевой строке ненулевые члены с размерностями $\leqslant n$ остаются ненулевыми во всех членах спектральной последовательности, в том числе в $E_{\infty}$. Следовательно, они останутся ненулевыми и в $H_{G}^{*}\left(X, Z_{p}\right)$. Теорема 25 доказана.

Изложенные в этом пункте соображения показывают, что в теоремах 19 и 20 не обязательно требовать тривиальность групп когомологий, а достаточно, чтобы выполнялось условие $\underline{\operatorname{ind}}_{G} X \geqslant n$. В теореме 20 надо также потребовать, чтобы отображение $f^{*}: H^{i}\left(M, Z_{p}\right) \rightarrow H^{i}\left(X, Z_{p}\right)$ было тривиальным при всех положительных $i$. В работе [35] приведены другие варианты понятия индекса $\left(Z_{p}\right)^{k}$-пространств, которые могут быть использованы для установления аналогов теорем типа Борсука-Улама. 
3.6. $\left(Z_{p}\right)^{k}$-действие в задаче Кнастера. Приведем обзор результатов по задаче Кнастера [36].

ГиПотезА 2 (Кнастер, 1947). Для всякой непрерывной бункции $f$ на единичной сфере $S^{d-1} \subset \mathbb{R}^{d}$ и всякого набора из $d$ точек $x_{1}, \ldots, x_{d} \in S^{d-1}$ найдется вращение $\rho \in S O(d)$ такое, что

$$
f\left(\rho\left(x_{1}\right)\right)=\cdots=f\left(\rho\left(x_{d}\right)\right) .
$$

В [37] было доказано, что для достаточно больших $d$ (примерно $d \geqslant 10^{12}$ ) эта гипотеза неверна, в [38] были построены контрпримеры к этой гипотезе для всех $d \geqslant 67$.

Тем не менее, есть и положительные результаты по гипотезе Кнастера для специальных конфигураций точек. В [39] было доказано, что гипотеза Кнастера верна для множества, состоящего из концов векторов ортонормированного базиса, в [40] гипотеза Кнастера доказана для любых конфигураций при $d=3$.

В работе Макеева [41] и других его работах были применены $Z_{p}$-эквивариантные отображения. Фактически гипотеза Кнастера была доказана для наборов из $p$ точек на сфере, группа симметрий которых содержит $Z_{p}$, для наборов из $p+1$ точки с $Z_{p}$-симметрией и для “почти $Z_{p}$-симметричных" наборов при $p \geqslant$ $2 d-2$ (в частности, для множества из 4 вершин правильного пятиугольника).

В [26] гипотеза Кнастера была доказана для конфигураций из $p^{k}$ точек с транзитивной группой симметрий $\left(Z_{p}\right)^{k}$, действие которой в пространстве задается перестановками базисных векторов, также из соображений непрерывности и гомотопического продолжения (см. п. 4.3) гипотеза Кнастера была доказана для конфигураций из $p^{k}+1$ точки с $\left(Z_{p}\right)^{k}$-симметрией.

Как в [41], так и в [26] доказательство гипотезы Кнастера по сути сводилось к следующему. Пусть группа симметрий $G=\left(Z_{p}\right)^{k}$ вложена в $S O(d)$. Тогда $S O(d)$ становится $G$-пространством. Отображение $f$ из формулировки гипотезы Кнастера дает $G$-эквивариантное отображение $g: S O(d) \rightarrow \mathbb{R}^{d}$, задаваемое формулой

$$
g: \rho \mapsto\left(\rho\left(x_{1}\right), \ldots, \rho\left(x_{d}\right)\right) .
$$

Нужно доказать, что образ этого отображения пересекает диагональ в $\Delta \subset \mathbb{R}^{d}$, где $\Delta=\{(t, t, \ldots, t)\}_{t \in \mathbb{R}}$.

Обозначим фактор $\mathbb{R}^{d} / \Delta$ через $W$ и рассмотрим на нем действие $G$. Как представление группы $G$ пространство $W$ не имеет тривиальных слагаемых (т. е. $\left.W^{G}=0\right)$. Ему соответствует $G$-эквивариантное расслоение $\eta: W \times S O(d) \rightarrow$ $S O(d)$, и препятствием к существованию введенного отображения $g$ является эквивариантный класс Эйлера расслоения $\eta$.

Класс Эйлера этого расслоения не равен нулю в $A_{G}$, Значит, надо только доказать, что естественное отображение $A_{G} \rightarrow H_{G}^{*}\left(S O(d), Z_{p}\right)$ инъективно в размерности $d-1$. Последнее доказывается рассмотрением спектральной последовательности из теоремы 24 с учетом того, что дифференциалы в этой последовательности должны отображать мультипликативные образующие алгебры $H^{*}\left(S O(d), Z_{p}\right)$ в классы Понтрягина и Эйлера соответствующего главного $S O(d)$-расслоения над $B G$ (т. е. все они трансгрессивны). 
В работе [42] доказывается гипотеза Кнастера для конфигурации из $p^{2}$ точек на некоторой окружности, расположенных в вершинах правильного многоугольника. В этой работе используется вычисление класса Эйлера соответствующего расслоения в $K$-теории, который оказывается ненулевым.

Кнастер сформулировал также более общий вариант своей задачи [36].

ГиПотезА 3 (обобщенная задача Кнастера, 1947). Для всякого непрерывного отображения $f: S^{d-1} \subset \mathbb{R}^{m}$ и всякого набора из $n=d-m+1$ точек $x_{1}, \ldots, x_{n} \in S^{d-1}$ найдется вращение $\rho \in S O(d)$ такое, что

$$
f\left(\rho\left(x_{1}\right)\right)=\cdots=f\left(\rho\left(x_{n}\right)\right) .
$$

Случай $m=d-1$ в этой гипотезе был ранее доказан в [43]. В работе [26] доказан частный случай этой гипотезы: $m=2, n=p^{k}$, в $\mathbb{R}^{n+1}$ есть некоторый базис $\left\{e_{1}, \ldots, e_{n}, e_{n+1}\right\}$ такой, что на множестве точек $\left\{x_{i}\right\}_{i=1}^{n}=\left\{e_{i}\right\}_{i=1}^{n}$ транзитивно действует группа $G=\left(Z_{p}\right)^{k} \subset S O(d+1)$.

Контрпримеры к обобщенной гипотезе Кнастера были найдены еще раньше, чем к гипотезе Кнастера для функций. В работах [44], [45] было замечено, что если конфигурация из $n$ точек содержится в некотором линейном подпространстве $L \subseteq \mathbb{R}^{d}$ размерности $l$ и выполняется неравенство

$$
\frac{l(2 n-l-1)}{2}<m(n-1),
$$

то обобщенная гипотеза Кнастера неверна из соображений размерности для почти всех таких конфигураций. В [46] показано, что обобщенная гипотеза Кнастера неверна для специально построенных конфигураций точек и функций при любых $d-1>m>2$. В [38] построены контрпримеры к обобщенной задаче Кнастера при $m=2$ и всех $d \geqslant 5$, а также получены новые контрпримеры в случае $m=1$ (обычная задача Кнастера) при $d=61,63,65$ и всех $d \geqslant 67$.

\section{7. Теория Люстерника-Шнирельмана для функций с симметри-} ями. Понятие индекса $\left(Z_{p}\right)^{k}$-пространств имеет также важное значение в теории Люстерника-Шнирельмана для критических точек функций с группой симметрий $G=\left(Z_{p}\right)^{k}$. Следуя книге [29], введем понятие эквивариантной категории Люстерника-Шнирельмана (в книге рассматривается более общее определение, чем приведенное здесь).

ОПРедЕЛЕНИЕ 13. Пусть $X-G$-пространство, тогда $G$-категорией $X$ назовем минимальный размер $G$-инвариантного покрытия $\left\{X_{1}, \ldots, X_{n}\right\}$ пространства $X$, в котором каждое вложение $\iota_{i}: X_{i} \rightarrow X \quad G$-гомотопно вложению некоторой орбиты $\iota: G / H \rightarrow X$. Будем обозначать $G$-категорию $G$-cat $X$.

Определенная таким образом категория оценивает снизу число $G$-орбит критических точек $G$-инвариантной $C^{2}$-гладкой функции $f$ на многообразии $X$, если функция $f$ гладкая класса $C^{2}$, собственная и ограничена либо сверху, либо снизу.

На самом деле $G$-категория, как правило, оценивается снизу через $G$-род (род в смысле Шварца) пространства [22]. 
ОПРЕДЕЛЕНИЕ 14 . Пусть $X-G$-пространство, тогда $G$-родом $X$ назовем минимальное $n$, для которого существует $G$-отображение $X \rightarrow G / H_{1} * G / H_{2} * \cdots *$ $G / H_{n}$, где $G / H_{i}$ изоморфны некоторым орбитам $G$ на $X$. Будем обозначать $G$-род $G$-gen $X$.

Основные результаты в этом направлении формулируются следующим образом [22], [29].

Теорема 26. Если конечная группа $G$ действует на $X$ свободно, то

$$
G \text {-gen } X \geqslant \overline{\operatorname{ind}}_{G} X+1 \text {. }
$$

ТеОрема 27. Пусть $G=\left(Z_{p}\right)^{k}$, тогда для любого $G$-пространства $X$ без неподвижных точек

$$
G \text {-gen } X \geqslant\left\lceil\frac{\underline{\text { ind }}_{G} X}{2}\right\rceil+1,
$$

причем если $p=2$, то

$$
G \text {-gen } X \geqslant \underline{\operatorname{ind}}_{G} X+1 .
$$

В работе [47] (см. также [35]) введен еще один численный индекс $\left(Z_{p}\right)^{k}$-действия $i_{G}(X)$, определяемый через спектральную последовательность теоремы 24 и обладающий свойством $i_{G}(X) \leqslant \underline{\operatorname{ind}}_{G} X+1$. Этот индекс оценивает $G$-род следующим образом.

ТеОРема 28. Пусть $G=\left(Z_{p}\right)^{k}$, тогда для любого компактного $G$-пространства $X$ без неподвижных точек

$$
G \text {-gen } X \geqslant i_{G}(X)
$$

Заметим, что теорема Люстерника-Шнирельмана о покрытии в терминах категории (или рода) формулируется так: для антиподального действия $G=Z_{2}$ на сфере $S^{n}$ выполнены неравенства $G$-cat $S^{n} \geqslant G$-gen $S^{n} \geqslant n+1$.

\section{4. Топологические методы и обобщения классических теорем комбинаторной геометрии}

4.1. Топологические методы оценки хроматического числа графов и гиперграфов. Обозначим множество всех $k$-элементных подмножеств $[n]$ через $\left(\begin{array}{c}{[n]} \\ k\end{array}\right)$.

ОПРЕДЕЛЕНИЕ 15. Графом Кнезера $K G_{n}^{k}$ называется граф, вершинами которого являются множества из $\left(\begin{array}{c}{[n]} \\ k\end{array}\right)$, причем два подмножества соединены ребром тогда и только тогда, когда они не пересекаются.

ОПРЕДЕЛЕНИЕ 16. Хроматическим числом графа $G$ называется минимальное количество цветов, в которые надо покрасить вершины графа, чтобы концы каждого ребра были покрашены в разные цвета (правильная раскраска). Хроматическое число обозначается $\chi(G)$. 
ОПредЕЛЕние 17. Гиперграфом на вершинах $V$ называется множество $V$ и некоторое семейство $\mathscr{F}$ непустых подмножеств $V$ (ребер или гиперребер). Гиперграф называется $k$-гиперграфом, если все множества семейства $\mathscr{F}$ имеют размер $k$. Часто гиперграф будет обозначаться множеством своих ребер, если понятно, какое множество является множеством вершин.

ОпредЕЛЕНиЕ 18. Для гиперграфа $\mathscr{F}$ на вершинах $V$ и непустого подмножества $W \subseteq V$ индуцированным гиперграфом $\mathscr{F}[W]$ называется гиперграф с вершинами $W$ и ребрами $\{F \in \mathscr{F}: F \subseteq W\}$.

ОПРЕДЕЛЕНИЕ 19. Хроматическим числом гиперграфа называется минимальное количество цветов, в которые нужно покрасить его вершины, чтобы ни одно из его ребер не было одноцветным. Будем обозначать хроматическое число $\chi(\mathscr{F})$.

Следующее утверждение было сформулировано в [48] и доказано в [49].

Теорема 29 (Ловас, 1978). Если $n \geqslant 2 k-1$, mo

$$
\chi\left(K G_{n}^{k}\right)=n-2 k+2 .
$$

Оценка сверху (построение раскраски) в этой теореме доказывается просто, оценка же снизу потребовала применения топологических методов, в одном из вариантов была применена обобщенная теорема Борсука-Улама для $Z_{2}$-действия. Этот результат важен в теории графов потому, что оценка снизу хроматического числа графа Кнезера с помощью линейного программирования, известная как "fractional chromatic number", дает $n / k$, что очень сильно отличается от настоящего хроматического числа.

В работе [50] было приведено более геометрическое доказательство теоремы 29, основанное на вложении конфигурации вершин в $\mathbb{R}^{n-2 k+2}$ и применении теоремы Борсука-Люстерника-Шнирельмана. В [51] было использовано вложение в $\mathbb{R}^{n-2 k+1}$ и теорема 29 была обобщена следующим образом.

ОПРЕДЕЛЕНиЕ 20. Кнезеровский граф $K G(\mathscr{F})$ гиперграфа $\mathscr{F}$ имеет множество вершин, совпадающее с семейством $\mathscr{F}$, две вершины соединены ребром тогда и только тогда, когда соответствующие гиперребра $F_{1}, F_{2} \in \mathscr{F}$ не пересекаются.

ОПРЕДЕЛЕНиЕ 21. Хроматическим дефектом гиперграфа $\mathscr{F}$ на множестве вершин $V$ называется число

$$
\operatorname{cd}_{m}(\mathscr{F})=\min \{|X|: \chi(\mathscr{F}[V \backslash X]) \leqslant m\} .
$$

Теорема 30 (Дольников, 1981). Для всякого гиперграфа Я્F имеем

$$
\chi(K G(\mathscr{F})) \geqslant \operatorname{cd}_{2}(\mathscr{F}) .
$$

Доказательство этого утверждения использует частный случай теоремы Дольникова о трансверсалях, подробнее см. п. 5.3. Оценка $\operatorname{cd}_{2}\left(\left(\begin{array}{c}{[n]} \\ k\end{array}\right)\right)=n-$ $2 k+2$ очевидна.

Оригинальное доказательство теоремы 29 было основано на следующем утверждении. 
ОПРЕДЕЛЕНИЕ 22. Для графа $G=(V, E)$ обозначим $\mathscr{N}(G)$ симплициальный комплекс с вершинами $V$ и симплексами, соответствующими наборам вершин, имеющим общего соседа. Соответствующее англоязычное название "neighborhood complex".

Tеорема 31 (Ловас, 1978). Eсли $\widetilde{H}^{i}\left(\mathscr{N}(G), Z_{2}\right)=0$ nрu $i \leqslant k-1$, mo $\chi(G) \geqslant$ $k+2$.

Естественно, в основе доказательства этой теоремы лежит некоторое $Z_{2}$-действие и его индекс. Изложим соответствующие рассуждения более подробно, следуя работе [52].

ОПРЕДЕЛЕНИЕ 23. Для графа $G=(V, E)$ обозначим $\mathscr{B}(G)$ симплициальный комплекс с вершинами, состоящими из двух копий $V_{1}$ и $V_{2}$ множества $V$, и симплексами, соответствующими парам $W_{1} \subseteq V_{1}, W_{2} \subseteq V_{2}$ таким, что каждое из множеств $W_{1}$ и $W_{2}$ имеет общего соседа и любые две вершины $w_{1} \in W_{1}$ и $w_{2} \in W_{2}$ соединены в $G$. Соответствующее англоязычное название "box complex".

Комплекс $\mathscr{B}(G)$ функториален относительно $G$ и имеет естественное $Z_{2}$-действие перестановками пар подмножеств. Для полного графа $K_{n}$ на $n$ вершинах комплекс $\mathscr{B}\left(K_{n}\right)$ гомотопически эквивалентен $(n-2)$-мерной сфере с антиподальным действием $Z_{2}$. Эти и некоторые другие соображения позволяют доказать следующую теорему [52].

Теорема 32 (Алон, Франкль, Ловас, 1986). Комплекс $\mathscr{B}(G)$ гомотопически эквивалентен $\mathscr{N}(G)$ и

$$
\chi(G) \geqslant \underline{\operatorname{ind}}_{Z_{2}} \mathscr{B}(G)+2 .
$$

В книге Козлова [53] понятие комплекса $\mathscr{B}(G)$ было обобщено: введено понятие полиэдрального комплекса гомоморфизмов графов $\operatorname{Hom}(G, H)$, вершинами которого являются собственно гомоморфизмы графов. Всякое отображение (раскраска) $G \rightarrow K_{n}$ индуцирует отображение $\operatorname{Hom}(H, G) \rightarrow \operatorname{Hom}\left(H, K_{n}\right)$ для любого графа $H$, при этом описанный выше комплекс $\mathscr{B}(G)$ с точки зрения оценки хроматического числа эквивалентен комплексу $\operatorname{Hom}\left(K_{2}, G\right)$ с действием группы $Z_{2}$ перестановками двух вершин $K_{2}$. Рассматривая другие графы $H$ с $Z_{2}$-действием, Козлов получает новые оценки хроматического числа в терминах $Z_{2}$-индекса соответствующих Нот-комплексов.

В работе [52] также были введены аналоги комплекса $\mathscr{B}(G)$ для гиперграфов и понятие графа Кнезера и его хроматического числа было обобщено следующим образом.

ОПРЕДЕЛЕниЕ 24. Для всякого гиперграфа $\mathscr{F}$ кнезеровский $r$-гиперграф $K G_{r}(\mathscr{F})$ построен на $\mathscr{F}$ как на вершинах, а его ребра - это наборы из $r$ попарно непересекающихся подмножеств $F_{1}, F_{2}, \ldots, F_{r} \in \mathscr{F}$.

Для системы $\mathscr{F}=\left(\begin{array}{c}{[n]} \\ k\end{array}\right)$ в [52] доказано следующее утверждение.

Теорема 33 (Алон, Франкль, Ловас, 1986). Положим $\mathscr{F}=\left(\begin{array}{c}{[n]} \\ k\end{array}\right)$. Если $n \geqslant$ $(m-1)(r-1)+r k, m o$

$$
\chi\left(K G_{r}(\mathscr{F})\right)>m
$$


В работах [54], [55] было установлено обобщение результата Дольникова.

Теорема 34 (Крыж, 1992-2000). Для всякого гиперграфа $\mathscr{F}$

$$
\chi\left(K G_{r}(\mathscr{F})\right) \geqslant \frac{1}{r-1} \operatorname{cd}_{r}(\mathscr{F}) .
$$

Все эти результаты существенно используют топологические соображения, в том числе теоремы типа Борсука-Улама для $Z_{p}$-действия.

В работе [56] эти результаты и их обобщения доказываются "комбинаторно", однако на самом деле просто формулируется соответствующий аналог леммы Таккера для $Z_{p}$-действия на симплициальных комплексах, вычисляются когомологии, но все рассуждения проводятся без явного использования топологических пространств.

В работе [57] рассматриваются комплексы гомоморфизмов $q$-гиперграфов $\left(q=p^{k}\right)$ с $\left(Z_{p}\right)^{k}$-действием и формулируются некоторые нижние оценки хроматического числа гиперграфов в терминах индексов соответствующих $\left(Z_{p}\right)^{k}$-пространств.

\section{2. Теорема Тверберга, “цветные” теоремы Каратеодори и Хелли.}

Теорема 35 (Тверберг [58], 1966). Пусть конечное множество $X \in \mathbb{R}^{d}$ состоит из $(d+1)(r-1)+1$ точек. Тогда $X$ можно разбить на $r$ множеств $X_{1}, \ldots, X_{r}$, выпуклые оболочки которых имеют общую точку.

Теорема Тверберга одновременно обобщает теорему Радона и дискретную теорему о центральной точке.

Один из самых простых способов доказать теорему Тверберга - использование "цветной” теоремы Каратеодори из [59].

Теорема 36 (цветная теорема Каратеодори; Барани, 1982). Пусть дано $d+1$ конечное множество $X_{1}, \ldots, X_{d+1}$ в $\mathbb{R}^{d}$. Тогда для всякой точки $x \in$ $\bigcap_{i=1}^{d+1} \operatorname{conv} X_{i}$ найдется система представителей $x_{i} \in X_{i}$, для которой

$$
x \in \operatorname{conv}\left\{x_{1}, \ldots, x_{d+1}\right\} .
$$

Также в работе [59] со ссылкой на Ловаса была приведена “цветная" теорема Хелли.

Теорема 37 (цветная теорема Хелли; Барани, Ловас, 1982). Пусть в $\mathbb{R}^{d}$ дано $d+1$ конечное непустое семейство выпуклых множеств $\mathscr{F}_{1}, \ldots, \mathscr{F}_{d+1}$. Если для любой системы представителей $C_{i} \in \mathscr{F}_{i}$ пересечение $\bigcap_{i=1}^{d+1} C_{i}$ непусто, то для некоторого $i$ пересечение семейства $\bigcap_{X \in \mathscr{F}_{i}} X$ непусто.

В контексте цветных теорем Каратеодори и Хелли была сформулирована следующая гипотеза.

ГиПотезА 4 (цветная теорема Тверберга; Барани). Предположим, что конечное множество $X$ из $r(d+1)$ точки в $\mathbb{R}^{d}$ раскрашено в $d+1$ ивет так, что точек каждого ивета ровно $r$. Тогда $X$ можно разбить на $r$ полноцветных подмножеств $X_{1}, \ldots, X_{r}$ из $d+1$ точки каждое так, что $\bigcap_{i=1}^{r} \operatorname{conv} X_{i} \neq \varnothing$. 
В работе [60] приведено доказательство этой гипотезы для $r=2$ со ссылкой на Ловаса, а также эта гипотеза была доказана для $d=2$ с помощью чисто геометрического рассуждения. В п. 4.4 будут обсуждаться топологические доказательства ослабленных вариантов этой гипотезы.

4.3. Интерпретация теорем существования в терминах препятствий и относительный класс Эйлера. Как уже было указано, некоторые геометрические теоремы существования можно сформулировать в терминах существования нулей сечения некоторого (эквивариантного) векторного расслоения. Основной способ убедиться в наличии нулей сечения векторного расслоения - доказать, что класс Эйлера расслоения не равен нулю.

Вычисление класса Эйлера может быть основано на явном описании когомологий, как это делается для $G$-эквивариантных расслоений, индуцированных из представлений группы $G$ (см. пп. 3.5 и 3.6). Однако есть и более простой способ вычислить класс Эйлера, основанный на следующем наблюдении.

Лемма 1. Пусть $X$ - n-мерное замкнутое гладкое многообразие, $\pi_{V}$ : $V \rightarrow X-k$-мерное векторное расслоение. Если $X$ и $V$ ориентируемь, то используем коэффичиенты $A=\mathbb{Z}$, иначе $A=Z_{2}$. Тогда для общего сечения $s: X \rightarrow V$ многообразие нулей сечения двойственно по Пуанкаре классу Эйлера $e(V)$.

Лемма 1 дает удобный способ вычисления класса Эйлера, если $k=n$. Тогда надо выбрать сечение расслоения $V$, которое имело бы только простые нули, что по определению означает: $s(X)$ трансверсально нулевому сечению $s_{0}(X)$. В случае $A=Z_{2}$ класс Эйлера соответствует четности количества этих нулей. Если же $A=\mathbb{Z}$, то для всякого нуля надо локально представить сечение в правильно ориентированных координатах $\left(x_{1}, \ldots, x_{n}\right)$ на $X$ и $\left(y_{1}, \ldots, y_{n}\right)$ на слое $V$ в виде

$$
y_{i}=\sum a_{i j} x_{j}+o\left(\sqrt{x_{1}^{2}+\cdots+x_{n}^{2}}\right)
$$

и назначить такому нулю индекс $\operatorname{sgn} \operatorname{det}\left(a_{i j}\right)$. Тогда класс Эйлера $V$ соответствует сумме индексов всех нулей. Этот метод называется методом тестовых сечений.

В приложении к конкретным геометрическим задачам метод тестовых сечений позволяет рассмотреть какой-то конкретный экземпляр задачи, для которого все нули сечения легко описываются, убедиться, что класс Эйлера ненулевой, и сделать отсюда вывод о существовании нулей во всех экземплярах задачи. Этот метод широко применяется в задачах о вписывании/описывании (см. п. 4.6) и в некоторых других случаях (см. п. 5.2).

Многообразие в методе тестовых сечений может быть некомпактно. Тогда класс Эйлера следует рассматривать в когомологиях с компактным носителем, но он будет зависеть не только от расслоения, но и от поведения сечения "на бесконечности". Для уточнения этих соображений полезно ввести понятие относительного класса Эйлера.

В первую очередь следует заметить, что относительный класс Эйлера относится не только к расслоению, но и к выбору конкретного сечения. Далее в этом 
пункте, если не оговорено иначе, для ориентируемых расслоений рассматриваются когомологии с целыми коэффициентами, если же расслоение может быть неориентируемым, то рассматриваются когомологии с коэффициентами в $Z_{2}$. Обозначение кольца коэффициентов опускается.

ОПРедЕЛЕНИЕ 25. Рассмотрим пару пространств $Y \subseteq X$ и $m$-мерное векторное расслоение $\pi_{V}: V \rightarrow X$. Пусть также задано не обращающееся в нуль сечение $s$ расслоения $V$ над $Y$. Назовем такую конструкцию $(V, s)$ частичным сечением.

В некоторых случаях будем считать, что сечение $s$ как-то продолжено на все пространство $X$. Естественно, в этом случае оно может обращаться в нуль на $X \backslash Y$. Но любые два продолженных сечения можно соединить между собой гомотопией, при которой гомотопия не будет менять сечение над $Y$.

Нетрудно доказать, что частичные сечения над парой $(X, Y)$ гомотопически классифицируются отображениями пары $(X, Y)$ в пару $(B O(m), B O(m-1))$, или $(B S O(m), B S O(m-1))$ в случае ориентируемых расслоений.

Для $(B O(m), B O(m-1))$ существует следующая явная конструкция. Рассмотрим каноническое $m$-мерное векторное расслоение $\gamma \rightarrow B O(m)$. Тогда паpa $(B(\gamma), S(\gamma))$ является одной из возможных реализаций $(B O(m), B O(m-1))$. Изоморфизм Тома показывает, что существует $u \in H^{m}(B O(m), B O(m-1))$ такой, что умножение элементов $H^{*}(B O(m))$ на $u$ дает изоморфизм когомологий с коэффициентами в $Z_{2}$

$$
H^{*}(B O(m), B O(m-1))=u H^{*}(B O(m)) .
$$

Такая же ситуация имеет место для пары $(B S O(m), B S O(m-1))$ и когомологий с целыми коэффициентами.

ОПРЕДЕЛЕНИЕ 26. Образ класса Тома $u \in H^{m}\left(B O(m), B O(m-1), Z_{2}\right)$ в $H^{m}\left(X, Y, Z_{2}\right)$ будем называть классом Эйлера по модулю 2 частичного сечения. В ориентируемом случае образ класса Тома $u \in H^{m}(B S O(m), B S O(m-1), \mathbb{Z})$ в $H^{m}(X, Y, \mathbb{Z})$ будем называть классом Эйлера частичного сечения. Будем обозначать класс Эйлера частичного сечения $e(V, s)$.

Следующая лемма следует из определений классов Тома и Эйлера. Несвязное объединение топологических пространств $X$ и $Y$ обозначим $X \sqcup Y$.

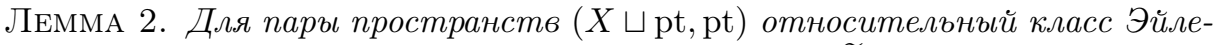
ра расслоения $V \rightarrow X \sqcup \mathrm{pt}$ с любым сечением над pt в $\widetilde{H}^{*}(X \sqcup \mathrm{pt}, \mathrm{pt})=H^{*}(X)$ совпадает с обычным классом Эйлера ограничения этого расслоения на $X$.

Из определения ясно, что класс Эйлера является первым препятствием (возможно, по модулю 2) к продолжению частичного ненулевого сечения до полного ненулевого сечения. Именно это свойство относительного класса Эйлера и нужно в геометрических приложениях.

ОПРЕДЕЛЕНИЕ 27. Пусть над $\left(X_{1}, Y_{1}\right)$ дано частичное сечение $\left(V, s_{1}\right)$, а над $\left(X_{2}, Y_{2}\right)$ дано частичное сечение $\left(W, s_{2}\right)$. Будем считать сечения $s_{i}$ продолженными на $X_{i}$. Тогда в произведении расслоений $V \times W$ сечение $s_{1} \times s_{2}$ дает 
частичное сечение над парой $\left(X_{1} \times X_{2},\left(X_{1} \times Y_{2}\right) \cup\left(Y_{1} \times X_{2}\right)\right)$. Назовем эту операцию произведением частичных сечений.

Следующая лемма выводится из рассмотрения классифицирующих пространств и мультипликативности класса Тома.

Лемма 3. Имеет место формула

$$
e\left(V \times W, s_{1} \times s_{2}\right)=e\left(V, s_{1}\right) \times e\left(W, s_{2}\right) .
$$

В частности, если $X_{1}=X_{2}=X$, то для пары частичных сечений $s_{1}$ и $s_{2}$ над $\left(X, Y_{1}\right)$ u $\left(X, Y_{2}\right)$ имеем

$$
e\left(V \oplus W, s_{1} \oplus s_{2}\right)=e\left(V, s_{1}\right) e\left(W, s_{2}\right) \in H^{*}\left(X, Y_{1} \cup Y_{2}\right) .
$$

Перейдем к формулировке утверждения о двойственности для относительного класса Эйлера. Пара $(X, Y)$ является относительным многообразием, если $X$ и $Y$ - симплициальные комплексы, $Y$ является подкомплексом $X, X \backslash Y$ топологическое многообразие. В частности, пара из компактного гладкого многообразия и его края $(M, \partial M)$ является относительным многообразием. $n$-мерное относительное многообразие $(X, Y)$ ориентируемо, если есть класс $w \in H^{n}(X, Y, \mathbb{Z})$, который имеет ненулевой прообраз в группе $H^{n}\left(X, X \backslash x_{0}, \mathbb{Z}\right)$ для каждой $x_{0} \in X \backslash Y$.

Относительное многообразие $(X, Y)$ назовем гладким, если $X \backslash Y$ - гладкое многообразие. Собственно, мы рассматриваем только гладкие относительные многообразия. Частичное сечение (продолженное на все $X$ ) расслоения $V \rightarrow X$ назовем общим, если многообразие $s(X \backslash Y) \subset V$ трансверсально нулевому сечению $s_{0}(X \backslash Y)$.

Лемма 4. Пусть $(X, Y)$ - $n$-мерное гладкое относительное многообразие, $\pi_{V}: V \rightarrow X-k$-мерное векторное расслоение. Если $(X, Y)$ и $V$ ориентируемы, то используем коэффичиенты $A=\mathbb{Z}$, иначе $A=Z_{2}$. Тогда для общего частичного сечения $s: X \rightarrow V$ (продолженного на все $X)$ многообразие нулей сечения, как класс из $H_{n-k}(X \backslash Y)$, двойственно по Пуанкаре-Лефшецу классу Эйлера $e(V, s)$.

В относительном случае эта лемма также дает возможность вычислять относительный класс Эйлера методом тестовых сечений.

В качестве примера использования относительного класса Эйлера приведем доказательство теоремы Брауэра.

ДоКАЗАТЕЛЬСтво тЕОРемЫ БРАУЭРА. Рассмотрим непрерывное отображение шара $f: B \rightarrow B$. Если оно не имеет неподвижных точек, то отображение

$$
s(x)=f(x)-x
$$

дает частичное сечение тривиального расслоения над парой $\eta: B \times \mathbb{R}^{d} \rightarrow$ $(B, \partial B)$. Хотя само расслоение $\eta$ тривиально, мы покажем, что $e(\eta, s) \neq 0 \in$ $H^{d}(B, \partial B)$. Действительно, сечение $s(x)$ можно непрерывно деформировать в сечение $s^{\prime}(x)=-x$, при этом на $\partial B$ эта деформация не будет обращать сечение 
в нуль. Но у сечения $s^{\prime}$ ровно один невырожденный нуль, следовательно, класс Эйлера ненулевой. Теорема доказана.

Наличие гомологического препятствия (в виде класса Эйлера) позволяет построить практически эффективные алгоритмы для реализации геометрических теорем существования, что сделано, в частности, в работах [61], [62] именно для теоремы Брауэра.

Применение гомологических препятствий в вычислительных алгоритмах основано на методе гомотопического продолжения (англоязычный термин "hоmotopy continuation"), в основе которого лежит следующее утверждение.

ТЕОРема 38 (о гомотопическом продолжении). Пусть расслоение $V \rightarrow X$ имеет ненулевой класс Эйлера. Пусть также гомотопия $s_{t}$ связывает два сечения этого расслоения $s_{0}$ и $s_{1}$. Тогда в пространстве гомотопии $X \times[0,1]$ некоторая компонента множества нулей сечения $s_{t}$ пересекается и с $X \times\{0\}$, u $c X \times\{1\}$.

Для относительного класса Эйлера формулировка аналогична. В случае, если $X$ является многообразием размерности $n$, слои $V$ также имеют размерность $n$ и сечение $s_{t}$ находится в общем положении, утверждение теоремы говорит о том, что некоторый нуль $s_{0}$ связан гладкой кривой с некоторым нулем $s_{1}$ в пространстве $X \times[0,1]$. Это наблюдение позволяет построить эффективные вычислительные алгоритмы для нахождения некоторых нулей произвольного сечения $s_{1}$, если для некоторого фиксированного сечения $s_{0}$ все нули известны.

\section{4. Топологические теоремы типа Тверберга, обобщения теоремы} ван Кампена-Флореса. Теорему Тверберга можно сформулировать следующим образом: если $f: \Delta^{(d+1)(r-1)} \rightarrow \mathbb{R}^{d}-$ линейное отображение, то найдутся $r$ попарно непересекающихся граней $F_{1}, \ldots, F_{r}$ симплекса $\Delta^{(d+1)(r-1)}$, для которых $\bigcap_{i=1}^{r} f\left(F_{i}\right) \neq \varnothing$.

Обобщения теоремы Тверберга связаны с тем, что вместо $\Delta^{(d+1)(r-1)}$ можно взять другой симплициальный комплекс, а вместо линейного отображения - непрерывное, последний случай называется топологической теоремой типа Тверберга.

В частности, в цветной теореме (гипотезе) Тверберга рассматривается $(d+1)$ кратный джойн $r$-элементного множества $K=[r]^{* d+1}$, в теореме ван Кампена-Флореса рассматривается остов симплекса и непрерывное отображение.

ОПРЕДЕЛЕНИЕ 28. Если для комплекса $K$ верна линейная теорема Тверберга с параметрами $r, d$, то скажем, что $K \in \mathrm{LT}(r, d)$. Если верна топологическая теорема с теми же параметрами, то $K \in \mathrm{TT}(r, d)$.

Топологическая теорема Тверберга для $\Delta^{(d+1)(r-1)}$ и $r$ простого была доказана в [63]. Эта теорема и теорема ван Кампена-Флореса были обобщены в [24], [64] для степеней простых чисел.

Теорема 39 (Барани, Шлосман, Сыч, Воловиков, 1981-1996). Ecли r - cmeпень простого числа, то $\Delta^{(d+1)(r-1)} \in \mathrm{TT}(r, d)$. 
Теорема 40 (Саркариа, Воловиков, 1991-1996). Если $r$ - степень простого числа и для натуральных чисел $r, k, d$ выполняется неравенство

$$
d(r-1) \leqslant r k
$$

$m o \Delta_{k}^{r(k+2)-2} \in \mathrm{TT}(r, d)$.

Последняя теорема доказана для простых $r$ в работе [65]. В [64] она доказана для степеней простого числа и в более общей формулировке: рассматриваются отображения не только в $\mathbb{R}^{d}$, но и в произвольное $d$-мерное многообразие (с некоторым условием на тривиальность отображения когомологий). Кроме того, совпадения ищутся не только в наборах попарно непересекающихся граней, но и для наборов граней, никакие $j$ из которых не имеют общей точки (при некотором фиксированном $j$ ).

В работе [66] (см. также [67]) был доказан следующий ослабленный вариант цветной теоремы Тверберга.

Теорема 41 (Живалевич, Вречица, 1992). Пусть $K=[t]^{* d+1}, r$ - степень простого числа $u t \geqslant 2 r-1$. Тогда $K \in \mathrm{TT}(r, d)$.

На самом деле в работе [66] этот результат был доказан только для простых $r$, но с учетом результатов работы [24] доказательство проходит и для степеней простого числа. Это утверждение любопытно тем, что пока не придумано доказательство даже его линейного аналога, не использующее топологических соображений. Иначе говоря, топологические методы в этой теореме являются существенными.

Ограничение на наличие степени простого числа в топологических теоремах типа Тверберга пока не удалось снять, в частности, гипотеза о том, что $\Delta^{(d+1)(r-1)} \in \mathrm{TT}(r, d)$ для $r$, не являющихся степенью простого числа, остается открытой.

Кратко сформулируем основные идеи доказательства теорем типа Тверберга.

ОПРЕДЕЛЕНИЕ 29. Для симплициального комплекса $K$ на вершинах $V$ вырезанным $r$-кратным джойном назовем комплекс, множество вершин которого есть $[r] \times V$, а множество симплексов состоит из всевозможных множеств

$$
\{1\} \times \sigma_{1} \cup\{2\} \times \sigma_{2} \cup \cdots \cup\{r\} \cup \sigma_{r},
$$

где $\sigma_{1}, \ldots, \sigma_{r}$ - набор попарно непересекающихся симплексов из $K$. Вырезанный $r$-кратный джойн обозначается $K_{\Delta}^{* r}$.

Аналогично вместо запрета попарных пересечений симплексов можно наложить запрет на пересечения по $k$ штук, тогда получим $k$-вырезанный $r$-кратный джойн $K_{\Delta(k)}^{* r}$. Кроме того, можно определить вырезанные степени и джойны для топологических пространств.

Очевидно, на $r$-кратных джойнах перестановками копий множества вершин $V$ действует группа $G=\left(Z_{p}\right)^{k}$ (при $r=p^{k}$ ), на вырезанных джойнах это действие не имеет неподвижных симплексов. В сформулированных обозначениях включение $K \in \mathrm{TT}(r, d)$ будет выполняться, если не существует 
$G$-отображения $K_{\Delta}^{* r} \rightarrow\left(\mathbb{R}^{d}\right)_{\Delta(r)}^{* r}$, причем последнее пространство гомотопически эквивалентно сфере $(d+1)(r-1)$-мерного $G$-расслоения без тривиальных слагаемых. Таким образом, связь с вычислением класса Эйлера и индекса $G$-действия очевидна. В цитированных результатах нетривиальность класса Эйлера устанавливалась исходя из оценки индекса $\left(Z_{p}\right)^{k}$-пространства через связность этого пространства.

В некоторых публикациях используется не вырезанный $r$-кратный джойн симплициального комплекса, а вырезанное $r$-кратное произведение, определяемое аналогично. Несмотря на другую конструкцию, такая модификация приводит к тем же результатам.

4.5. Двойственные теоремы о центральной точке и Тверберга. Зафиксируем некоторые определения и обозначения, которые будут использоваться в этом пункте и в разделе 5 .

ОПРЕДЕЛЕНИЕ 30. Аффинное подмногообразие $\mathbb{R}^{d}$ размерности $k$ будем называть $k$-плоскостью, $(d-1)$-плоскость будем называть гиперплоскостью.

ОПРЕДЕЛЕНИЕ 31. Множество $k$-плоскостей, пересекающих данное множество $X \subseteq \mathbb{R}^{d}$, обозначим $I(X, k)$.

Приведем некоторые результаты работы автора [68].

Теорема 42 (Карасёв, 2008). Пусть в $\mathbb{R}^{d}$ дано семейство $\mathscr{F}$ из $n$ гиперплоскостей общего положения. Тогда найдется точка $x$ такая, что всякий проходящий через нее луч пересекает как минимум $\left\lfloor\frac{n+d}{d+1}\right\rfloor$ гиперплоскостей
из $\mathscr{F}$.

Под общим положением семейства гиперплоскостей в $\mathbb{R}^{d}$ подразумевается, что любые $d$ имеют ровно одну общую точку, а любые $d+1$ не имеют общей точки. В следующем утверждении используется параметризация пространства плоскостей из п. 5.2 .

ТЕОРема 43 (Карасёв, 2008). Пусть на многообразии гиперплоскостей $\gamma_{d}^{1}$ задана абсолютно непрерывная вероятностная мера $\mu$ с компактным носителем. Тогда найдется точка $x$ такая, что для всякого луча $r$ с началом в $x$

$$
\mu(I(r, d-1)) \geqslant \frac{1}{d+1} .
$$

ОПРЕДЕЛЕНИЕ 32 . Будем говорить, что $d+1$ гиперплоскость $h_{1}, \ldots, h_{d+1}$ общего положения в $\mathbb{R}^{d}$ образует симплекс $S$, если $S$ является выпуклой оболочкой множества точек $\left\{x_{i}\right\}$, определяемых как

$$
x_{i}=\bigcap_{j \neq i} h_{j}
$$

Очевидно, что каждая гипергрань $S$ лежит на соответствующей гиперплоскости $h_{i}$. 
ГиПотезА 5 (двойственная теорема Тверберга). Пусть в $\mathbb{R}^{d}$ дано семейство из $(d+1) n$ гиперплоскостей общего положения. Тогда их можно разбить на наборы из $d+1$ гиперплоскости так, что все симплексы, образованные наборами, имеют общую точку.

В работе автора [68] эта гипотеза сформулирована и доказана для $d=2$ и в следующем частном случае.

Теорема 44 (Карасёв, 2008). Пусть в $\mathbb{R}^{d}$ дано семейство из $(d+1) n$ гиперплоскостей общего положения, причем $n=p^{k}$, где $p$ - простое число.

Тогда эти гиперплоскости можно разбить на $n$ непересекающихся наборов из $d+1$ гиперплоскости так, что все симплексы, образованные наборами, имеют общую внутреннюю точку.

Также в этой работе доказана двойственная версия цветной теоремы Тверберга.

Tеорема 45 (Карасёв, 2008). Пусть в $\mathbb{R}^{d}$ дано семейство из $(d+1) t$ гиперплоскостей общего положения, где $t \geqslant 2 r-1, r=p^{k}, p$ - простое число. Пусть эти гиперплоскости разбиты на $d+1$ семейство (ивет) по $t$ элементов.

Тогда из данных гиперплоскостей можно выбрать $r$ непересекающихся наборов из $d+1$ гиперплоскости так, что все симплексы, образованные наборами, имеют общую внутреннюю точку и каждый набор разноцветный, т.е. не содержит пары одноцветных гиперплоскостей.

Доказательство двойственной теоремы о центральной точке основано на применении аналога теоремы Брауэра о неподвижной точке. Двойственные теоремы типа Тверберга доказываются с помощью построения препятствия в виде класса Эйлера. По сути построенное препятствие является прямым произведением препятствий из топологической (цветной) теоремы Тверберга и препятствия из теоремы Брауэра.

Также в работе [68] доказываются двойственные теоремы о центральной трансверсали (см. п. 5.5).

4.6. Теоремы существования для вписанных и описанных фигур. Приведем некоторые результаты о вписывании и описывании фигур.

ОПРЕДЕЛЕНИЕ 33. Многогранник $P$ подобно (гомотетично, конгруэнтно, аффинно) вписъвается в выпуклое тело $T$, если существует преобразование подобия (гомотетия, движение, аффинное преобразование) $\rho$, для которого все вершины $\rho(P)$ лежат на $\partial T$.

ОПРЕДЕЛЕНИЕ 34. Многогранник $P$ подобно (гомотетично, конгруэнтно, аффинно) описывается около выпуклого тела $T$, если существует преобразование подобия (гомотетия, движение, аффинное преобразование) $\rho$, для которого $\rho(P) \supseteq T$ и $T$ пересекает каждую гипергрань $\rho(P)$.

В задачах на вписывание многогранника удобно рассматривать гладкие тела, а в задачах на описывание многогранника - строго выпуклые. Более общие 
же результаты, как правило, получаются предельным переходом, но в некоторых случаях при предельном переходе не удается доказать, что последовательность вписанных/описанных фигур не стремится к вырождению.

Типичным примером является теорема Шнирельмана, в которой пока не известно, верна ли она для произвольной непрерывной замкнутой кривой без самопересечений (жордановой кривой). Если жорданова кривая в окрестности каждой точки является графиком непрерывной функции в некоторой системе координат, то предельный переход проходит, а в общем случае предельный переход может привести к вырождению вписанного квадрата. С другой стороны, в теореме Какутани предельный переход проходит всегда, так как куб не может выродиться в точку или устремиться к бесконечно большому кубу.

Доказательство теоремы Шнирельмана имеет любопытную историю. Собственно в статье Шнирельмана [15] доказательство было в принципе неверным. В работе [69] была сделана попытка исправить доказательство Шнирельмана, однако основная лемма о непрерывной зависимости вписанного квадрата от кривой принципиально неверна и к ней существуют простые контрпримеры. Также в [69] был приведен план доказательства вписываемости правильного кроссполитопа в произвольное гладкое выпуклое тело, однако этот план опирался на обобщаемость основной леммы на случай размерности $d \geqslant 3$.

В работе [70] было приведено в целом правильное доказательство теоремы Шнирельмана, однако автор ограничился рассмотрением случая аналитических кривых. Кроме того, в этой работе было приведено утверждение, являющееся частным случаем теоремы 38 (о гомотопическом продолжении), это утверждение показывает, что непрерывной зависимости вписанного квадрата от кривой нет, однако имеет место "гомотопически непрерывная" зависимость.

В работе [71] приведено доказательство теоремы Шнирельмана для случая простых замкнутых ломаных. Кроме того, доказательство теоремы Шнирельмана, основанное на методе тестовых сечений, известно Макееву. Для полноты изложения приведем доказательство теоремы Шнирельмана, основанное на идеях Макеева и изложенное более строго с использованием введенной выше терминологии.

ДокаЗАтеЛЬСтво теОРемы ШНИРеЛЬмАНА. Рассмотрим некоторую ориентированную гладкую кривую $C \in \mathbb{R}^{2}$.

Обозначим $X$ конфигурационное пространство наборов из четырех (возможно, совпадающих) точек $\left(p_{1}, p_{2}, p_{3}, p_{4}\right)$ на кривой таких, что они занумерованы в порядке обхода кривой. Обозначим $Y \subset X$ пространство вырожденных конфигураций, в которых некоторые точки совпадают.

Теперь построим отображение $f: X \rightarrow \mathbb{R}^{6}$, заданное покоординатно как

$$
\begin{array}{lll}
x_{1}=\left|p_{2}-p_{1}\right|, & x_{2}=\left|p_{3}-p_{2}\right|, & x_{3}=\left|p_{4}-p_{3}\right|, \\
x_{4}=\left|p_{1}-p_{4}\right|, & x_{5}=\left|p_{3}-p_{1}\right|, & x_{6}=\left|p_{4}-p_{2}\right| .
\end{array}
$$

Рассмотрим подпространство $L \in \mathbb{R}^{6}$, натянутое на векторы $(1,1,1,1,0,0)$ и $(0,0,0,0,1,1)$ и соответствующее факторпространство $V=\mathbb{R}^{6} / L$. Отображение $f$ факторизуется до отображения $g: X \rightarrow V$. 
Отображение $g$ перестановочно с действием группы $Z_{4}$. Действие $Z_{4}$ на $X$ задается циклическими перестановками точек $p_{i}$, действие на $V$ задается циклическими перестановками координат $x_{1}, \ldots, x_{4}$ и перестановками координат $x_{5}, x_{6}$ факторгруппой $Z_{4} / Z_{2}=Z_{2}$. Таким образом, $g$ можно рассматривать как сечение $Z_{4}$-расслоения. Понятно, что для доказательства наличия вписанного в $C$ квадрата надо доказать, что $g$ имеет нуль на $X \backslash Y$.

Прямое применение класса Эйлера невозможно, так как построенное сечение $g$ имеет нули на $Y$, соответствующие конфигурациям, в которых $p_{1}=p_{2}=$ $p_{3}=p_{4}$. Рассмотрим пространство $X_{\varepsilon} \subset X$, в котором расстояние (в смысле параметра на кривой) между каждой парой точек не менее $\varepsilon$, и его подпространство $Y_{\varepsilon}$, в котором расстояние между некоторыми парами равно $\varepsilon$. Из гладкости кривой $C$ следует, что для достаточно малых $\varepsilon$ сечение $g$ не имеет нулей на $Y_{\varepsilon}$. Имеет смысл относительный класс Эйлера $e(g) \in H_{Z_{4}}^{4}\left(X_{\varepsilon}, Y_{\varepsilon}, Z_{2}\right)$.

Заметим, что при непрерывной деформации кривой $C$, оставляющей ее гладкой, $\varepsilon$ из предыдущего абзаца можно выбрать одинаковым для всех кривых деформации. Следовательно, при деформации построенный класс Эйлера не изменяется. Кривую можно деформировать, сохраняя гладкость, в эллипс с разными полуосями. Легко проверить, что сечение $g$ в этом случае имеет ровно один невырожденный нуль на $\left(X_{\varepsilon} \backslash Y_{\varepsilon}\right) / Z_{4}$, следовательно, по лемме 4 класс Эйлера $e(g)$ не нулевой. Теорема доказана.

Следующая теорема Громова [72] существенно использует топологическую технику в доказательстве.

ТеОрема 46 (Громов, 1969). Пусть $\Delta \subset \mathbb{R}^{d}-d$-мерный симплекс, а компакт $T \subset \mathbb{R}^{d}$ имеет $C^{1}$-гладкую границу и ненулевую эйлерову характеристи$\kappa у \chi(T) \neq 0$. Тогда $\Delta$ положительно гомотетично вписывается в $T$.

В [73] доказано следующее обобщение теоремы Шнирельмана для трехмерного случая.

Теорема 47 (Макеев, 2003). Пусть $C$ - правилъный октаэдр в $\mathbb{R}^{3}$, а $T$ гладкое выпуклое тело. Тогда $C$ подобно вписывается в $T$.

Б. Грюнбаумом была сформулирована следующая гипотеза.

ОПРеДЕЛЕНиЕ 35. Для множества $S \in \mathbb{R}^{d}$ обозначим его разностное множество

$$
S-S=\{x-y: x, y \in S\} .
$$

Если $S$ - выпуклое множество, то $S-S$ - тоже выпуклое.

ГиПотеза 6 (Грюнбаум). Пусть $\Delta$ - симплекс в $\mathbb{R}^{d}, Q=\Delta-\Delta-$ разностное множество симплекса, а $T \subset \mathbb{R}^{d}-$ гладкое выпуклое тело. Тогда $Q$ аффинно вписывается в $T$.

В работе [74] эта гипотеза доказана для $d=3$. Доказательство основано на рассмотрении пространства всех возможных аффинных преобразований $\rho$, построения над ним расслоения, сечение которого должно обратиться в нуль для решения задачи, и вычислении эквивариантного класса Эйлера расслоения 
с помощью тестовых сечений. В [74] также были рассмотрены случаи негладких $T$.

Отметим связь между задачами об описывании и проблемой (гипотезой) Борсука.

ТЕОРема 48 (Пал, Эглстон [75], [76], 1920-1958). Всякий компакт $K \in \mathbb{R}^{d}$ может быть вложен в тело постоянной ширины, равной диаметру $K$.

В соответствии с предыдущим результатом если некоторый многогранник $P$ конгруэнтно описывается вокруг всякого выпуклого тела $T$ постоянной ширины 1 , то для решения проблемы Борсука достаточно разбить $P$ на части диаметра, меньшего 1. На самом деле для решения проблемы Борсука достаточно, чтобы всякое тело постоянной ширины $T$ можно было движением поместить в $P$, что, очевидно, следует из конгруэнтной вписываемости $T$ в $P$.

ОПределЕНиЕ 36 . Пусть $P$ - выпуклое тело в $\mathbb{R}^{d}$. Если для всякого тела $T$ постоянной ширины 1 в $\mathbb{R}^{d}$ найдется движение $\rho$ такое, что $\rho(P) \supseteq T$, то $P$ называется универсалъной покрышкой.

С помощью нахождения универсальных покрышек можно получать и конкретные оценки сверху на диаметр частей разбиения в проблеме Борсука.

В двумерном случае имеет место следующий результат [75].

ТеОрема 49 (Пал, 1920). Пусть $P$ - правильный шестиугольник в $\mathbb{R}^{2}$, $T \subset \mathbb{R}^{2}$ - фигура постоянной ширины. Тогда $P$ подобно описывается вокруг $T$.

В трехмерном случае есть аналогичный результат [77].

ТЕОРема 50 (Гейл, 1953). Пусть $P$ - правильный октаэдр с расстоянием между противоположными гранями, равными единице, $T$ - тело постоянной ширины 1 в $\mathbb{R}^{3}$. Тогда $P$ конгруэнтно описывается вокруг $T$.

В [74] исследовался вопрос о нахождении универсальных покрышек и была сформулирована следующая гипотеза, обобщающая результат из работы [75].

ГиПотезА 7 (Макеев, 1995). Пусть $D$ - многогранник, двойственный в $\mathbb{R}^{d}$ $\kappa$ разностному множеству правильного симплекса, $T \subset \mathbb{R}^{d}-$ тело постоянной ширины. Тогда D подобно описывается вокруг $T$.

В работе [78] (и независимо в работе [79]) эта гипотеза была доказана для $d=3$, в [78] было также доказано следующее утверждение.

Теорема 51 (Макеев, 1997). Пусть $D$ - многогранник, двойственный в $\mathbb{R}^{3}$ к разностному множеству правильного симплекса, $T$ - строго выпуклое тело. Тогда D аффинно описъвается вокруг $T$.

Доказательство гипотезы 7 для $d=3$ позволило доказать следующее усиление гипотезы Борсука в трехмерном случае: всякое множество диаметра 1 в $\mathbb{R}^{3}$ можно разбить на четыре части диаметра не более 0.98 . Это является наилучшим результатом для $\mathbb{R}^{3}$ на текущий момент.

Доказательство теоремы 51 фактически основано на вычислении класса Эйлера. Что касается бо́льших размерностей, в работе [79] было показано, что 
для $d \geqslant 4$ класс Эйлера в гипотезе 7 нулевой. Это значит, что простого топологического доказательства гипотезы 7 для $d \geqslant 4$ нет.

В работе автора [80] теорема Макеева о вписывании октаэдра обобщается следующим образом.

ОПРЕДЕЛЕНИЕ 37 . Пусть $\left(e_{1}, \ldots, e_{d}\right)$ - некоторый базис в $\mathbb{R}^{d}$. Kроссполитопом в $\mathbb{R}^{d}$ назовем выпуклую оболочку точек

$$
e_{1},-e_{1}, e_{2},-e_{2}, \ldots, e_{d},-e_{d}
$$

и все ее образы при движениях. Если базис ортогональный и длины всех векторов базиса равны, то будем говорить, что кроссполитоп правильный.

Теорема 52 (Карасёв, 2008). Предположим, что $H \subset \mathbb{R}^{d}$ - образ гладкого вложения сферы $\imath: S^{d-1} \rightarrow \mathbb{R}^{d}, d=p^{k}$ - степень простого числа, $C$ - правильный кроссполитоп в $\mathbb{R}^{d}$. Тогда $C$ подобно вписъвается в $\mathrm{H}$.

В работе [80] доказывается вариант этого утверждения, когда кроссполитоп $C$ не правильный, но имеет $\left(Z_{p}\right)^{k}$-симметрию, кроме того, там же рассматривались некоторые ослабления условия гладкости (в случае поверхности выпуклого тела) в этой теореме.

Доказательство теоремы сводится к вычислению относительного класса Эйлера в эквивариантных когомологиях. Показывается, что этот класс Эйлера является прямым произведением класса Эйлера теоремы Брауэра и класса Эйлера из доказательства Воловиковым гипотезы Кнастера для $\left(Z_{p}\right)^{k}$-действия (см. п. 3.6 и статью [26]).

Множество более специальных теорем о вписывании и описывании можно найти в диссертации Макеева [73], в которой также детально изучаются вопросы предельного перехода от "хороших" тел к произвольным и вырождения вписанных/описанных многогранников.

\section{7. Бильярды в выпуклых телах.}

ОПРЕДЕЛЕНИЕ 38. Периодическая бильярдная траектория в гладком выпуклом теле $T \in \mathbb{R}^{d}$ - это замкнутая ломаная $P \subset T$, все вершины которой находятся на границе $T$ и в каждой вершине направление ломаной меняется по закону упругого отражения.

ОПРедЕЛЕНИЕ 39. Пусть $P$ - периодическая бильярдная траектория. Количество вершин $P$ обозначим $n(P)$.

Ясно, что периодические траектории однозначно соответствуют наборам вершин $\left(x_{1}, x_{2}, \ldots, x_{n}\right)$, индексы можно рассматривать по модулю $n$. В наборе вершин надо запретить совпадения $x_{i}=x_{i+1}$.

Рассматривается следующая задача: найти оценки снизу количества различных бильярдных траекторий $P$ с данным $n(P)$ в $d$-мерном гладком выпуклом теле $T$. Под "различными траекториями" подразумеваются орбиты группы диэдра $D_{n}$, которая естественно действует на вершинах траекторий.

Первые оценки снизу найдены в работе [81] в случае $d=2$. 
ТЕОрема 53 (Биркгоф, 1927). В двумерной гладкой выпуклой фигуре найдется не менее $\varphi(n)$ разных бильярдных траекторий с $n(P)=n$.

Здесь $\varphi(n)$ - функция Эйлера.

В случае $n(P)=2$ и произвольной размерности $d$ в работе [82] найдена оценка снизу, равная $d$. Как показывает пример эллипсоида с разными осями, эта оценка точна.

Случай $d=3$ был рассмотрен в работе [83], но позже в ней была обнаружена ошибка, на что указано в [84], [85].

В работах [84], [85] были найдены оценки снизу для разных случаев.

Теорема 54 (Фарбер, Табачников, 2002). Пусть $T \subset \mathbb{R}^{d}-$ гладкое выпуклое тело, $d \geqslant 4$, а $n$ - нечетное число. Тогда в $T$ найдется не менее

$$
\left\lfloor\log _{2}(n-1)\right\rfloor+d-1
$$

разных бильярдных траекторий $P$ с $n(P)=n$.

Если $n$ простое, а d четное, то разных траекторий не менее $n$. Если $n$ простое, а д нечетное, то разных траекторий не менее $(n+1) / 2$.

В работе автора [33] для простых $n$ оценки усилены.

Теорема 55 (Карасёв, 2008). Пусть $T \subset \mathbb{R}^{d}-$ гладкое выпуклое тело, $d \geqslant 3$, а $n$ - простое число. Тогда в $T$ найдется не менее $(d-2)(n-1)+2$ разных билълрдных траекторий $P$ с $n(P)=n$.

Доказательство этой теоремы, как и результаты [84], [85], основано на сведении задачи к нахождению критических точек функции длины ломаной, далее делается оценка категории Люстерника-Шнирельмана через строение когомологий. При этом, в отличие от предыдущих работ, находится $Z_{p}$-индекс $(p=n)$ конфигурационного пространства и делается оценка эквивариантной категории Люстерника-Шнирельмана через индекс по теореме 26.

4.8. Деление мер на равные части и части заданной меры. В этом пункте будут рассматриваться задачи деления абсолютно непрерывных вероятностных мер в $\mathbb{R}^{d}$ на равные части или части заданной меры при помощи разбиений $\mathbb{R}^{d}$ специального вида.

В п. 2.4 уже формулировалась теорема о бутерброде, в работе [86] была поставлена задача о разрезании одной меры на равные части несколькими гиперплоскостями, которая в более общем виде сформулирована в [87].

ЗАДАчА 1 (Грюнбаум, Рамос, 1960-1996). Найти минимальное d, обладающее следующим свойством: для всякого набора из $j$ абсолютно непрерывных вероятностных мер в $\mathbb{R}^{d}$ найдутся $k$ гиперплоскостей, разбивающих каждую из этих мер на $2^{k}$ равньх частей. Минимальное такое $d$ обозначим $\Delta(j, k)$.

Теорема о бутерброде тогда может быть переформулирована так: $\Delta(d, 1)=d$. В случае деления одной меры предполагалось, что $\Delta(1, d)=d$, это утверждение было доказано для $d=3$ в [88], однако в [89] было показано, что при $d \geqslant 5$ выполнено неравенство $\Delta(1, d)>d$. Гипотеза $\Delta(1,4)=4$ пока остается 
открытой. Автором была предпринята попытка (не опубликовано) доказать эту гипотезу вычислением соответствующего эквивариантного относительного класса Эйлера. К сожалению, класс Эйлера оказался нулевым.

Оценка снизу получена рассмотрением меры, сосредоточенной на кривой моментов, т. е. параметрически заданной кривой

$$
\left(t, t^{2}, \ldots, t^{d}\right) \in \mathbb{R}^{d}, \quad t \in \mathbb{R} .
$$

Пересечения гиперплоскостей с кривой моментов - это произвольные наборы из $d+1$ точки на этой кривой и только они. Из этих соображений в работе [87] была установлена оценка снизу $\Delta(j, k) \geqslant j \frac{2^{k}-1}{k}$.

Также в [87] была сформулирована тривиальная оценка сверху $\Delta(j, k) \leqslant$ $j 2^{k-1}$ и, с помощью топологических методов, были доказаны некоторые нетривиальные оценки сверху, общие формулировки которых мы не приводим, так как они громоздки и требуют дополнительных определений и обозначений.

В работе [90] с помощью более тонкой техники доказываются новые оценки сверху чисел $\Delta(j, k)$. Общие формулировки результата также достаточно громоздки и требуют введения нескольких дополнительных обозначений, приведем лишь некоторые частные результаты этой работы.

Теорема 56 (Мани-Левицка, Вречица, Живалевич, 2006).

$$
\Delta\left(2^{m+1}-1,2\right)=3 \cdot 2^{m}-1, \quad \Delta\left(2^{m}+r, k\right) \leqslant 2^{k+m-1}+r .
$$

Результаты были получены с помощью вычисления препятствия как методом тестовых сечений, так и с помощью явных вычислений в $\left(Z_{2}\right)^{k}$-эквивариантных когомологиях.

ОПРЕДЕЛЕНИЕ 40. Систему из $k$ различных половинок гиперплоскостей в $\mathbb{R}^{d}$, имеющих общим краем плоскость коразмерности 2 , назовем невырожденным $k$-пропеллером. Общепринятое англоязычное обозначение $k$-fan.

Назовем $k$-пропеллер правильным, если он разбивает $\mathbb{R}^{d}$ на конгруэнтные части.

Заметим, что при рассмотрении неправильных $k$-пропеллеров одна из частей разбиения может быть невыпуклой. Также в некоторых задачах на разбиение меры требуется рассматривать вырожденные системы.

ОПРедЕЛЕНИЕ 41 . Вырожденным $k$-пропеллером называется система из $k$ параллельных гиперплоскостей.

Следующая теорема объединяет результаты из [91] и [73].

Теорема 57 (Макеев, 1994). Если выполняется неравенство $2 d-1 \geqslant$ $m(p-1)$ и $p>2$ - простое число, то

1) для любого набора из $m+1$ абсолютно непрерывной вероятностной меры в $\mathbb{R}^{d}$ найдется (возможно, неправильный или вырожденный) p-пропеллер, разбивающий каждую меру на равные части; 
2) для любого набора из $m$ абсолютно непрерывных вероятностных мер в $\mathbb{R}^{d}$ найдется правильный р-пропеллер, разбивающий каждую меру на равнье части.

Пункт 2) этой теоремы получается из пункта 1), если одну из мер взять равномерно распределенной на сфере радиуса $R$ и устремить $R$ к бесконечности. В [92] также получен результат о разбиении меры пропеллером на "почти равные" части.

Теорема 58 (Макеев, 1996). Для всякой абсолютно непрерывной вероятностной мерь $\mu$ на $\mathbb{R}^{2}$ найдется правильный 5-пропеллер, делящий плоскость на части $C_{1}, C_{2}, C_{3}, C_{4}, C_{5}$, для которьх

$$
\mu\left(C_{1}\right)=\mu\left(C_{2}\right)=\mu\left(C_{3}\right)=\mu\left(C_{4}\right) \geqslant \mu\left(C_{5}\right) .
$$

Разбиение мер пропеллером фактически представляет собой разбиение ортогональных проекций этих мер на некоторое двумерное пространство. В поиске аналогичных утверждений для проекции на $\mathbb{R}^{k}$ при $k>2$ в работе [93] была сформулирована следующая гипотеза.

ОПредЕлЕНиЕ 42. Пусть симплекс $S \in \mathbb{R}^{k}$ содержит внутри себя начало координат. Тогда набор из конических оболочек его гиперграней $C_{1}, \ldots, C_{k+1}$ называется простым коническим разбиением. Если симплекс $S$ правильный и начало координат совпадает с его центром, то простое коническое разбиение называется правилъным. Сдвиг простого конического разбиения на вектор $c$ будем называть простым коническим разбиением с иентром с. Соответствующий термин из работы [94] - "(regular) radial dissection".

ГиПотезА 8 (Вречица, Живалевич, 1992). Пусть в $\mathbb{R}^{d}$ дана $k+1$ абсолютно непрерывная вероятностная мера $\mu_{1}, \ldots, \mu_{k+1}$. Тогда для некоторой ортогональной проекции $\pi: \mathbb{R}^{d} \rightarrow L$ на $(d-k)$-мерное подпространство существует простое коническое разбиение $L$ на части $C_{1}, \ldots, C_{d-k+1}$ с некоторым иентром с $\in L$ такое, что для всех $i \in[k+1] u j \in[d-k+1]$

$$
\mu_{i}\left(\pi^{-1}\left(C_{j}\right)\right)=\frac{1}{d-k+1} .
$$

В частном случае $k=1$ этот результат следует из теоремы 64 (см. далее), в случае $k=d-2$ он следует из теоремы 57 , в случае $k=d-1$ - это просто теорема о бутерброде.

В работе [95] (см. также [96]) был доказан следующий результат о делении нескольких мер на отрезке на равные части.

Теорема 59 (Алон, 1987). Пусть на отрезке I заданы абсолютно непрерывные вероятностные меры $\mu_{1}, \ldots, \mu_{n}$. Для целого числа $r \geqslant 2$ положим $N=(n+1)(r-1)$. Тогда I можно разбить на $N+1$ отрезок $I_{1}, \ldots, I_{N+1}$, а семейство $\mathscr{F}=\left\{I_{i}\right\}_{i \in[N+1]}$ можно разбить на $r$ подсемейств $\mathscr{F}_{1}, \ldots, \mathscr{F}_{r}$ так, что для любых $i \in[n], j \in[r]$

$$
\mu_{i}\left(\bigcup \mathscr{F}_{j}\right)=\frac{1}{r} .
$$


Доказательство этой теоремы использует то же препятствие, что и доказательство топологической теоремы Тверберга. Но в этой теореме число $r$ не обязано быть простым, так как из случаев для $r=r_{1}$ и $r=r_{2}$ непосредственно выводится случай $r=r_{1} r_{2}$.

В работе [97] это утверждение обобщено на случай разбиения нескольких мер на $d$-мерном кубе следующим образом.

Теорема 60 (де Лонгевиль, Живалевич, 2008). Пусть $n, d, k$ - натуральные числа $и k \geqslant 2$. Предположим, что на кубе $I^{d}$ заданъ абсолютно непрерывные вероятностные меры $\mu_{1}, \ldots, \mu_{n}$. Рассмотрим набор натуралъных чисел $m_{1}, \ldots, m_{d}$ такой, что $m_{1}+\cdots+m_{d}=n(k-1)$.

Тогда найдется набор $\mathscr{H}$ из $n(k-1)$ гиперплоскостей в $\mathbb{R}^{d}$ со следующими свойствами. Для любого $i \in[d]$ ровно $m_{i}$ гиперплоскостей из $\mathscr{H}$ перпендикулярны $i$-й оси координат, семейство $\mathscr{H}$ дает разбиение $I^{d}$ на семейство параллелепипедов ЯР. Семейство Р्Р можно разбить на $k$ подсемейств $\mathscr{P}_{1}, \ldots, \mathscr{P}_{k}$ так, что для любых $i \in[n], j \in[k]$

$$
\mu_{i}\left(\bigcup \mathscr{P}_{j}\right)=\frac{1}{k} .
$$

Следующее утверждение о разбиении меры конусами на равные части при $d=3$ было доказано Макеевым [98], приведенная здесь формулировка получена автором.

Теорема 61 (Макеев, Карасёв, 1988-2008). Пусть $G=\left(Z_{p}\right)^{k}, d=p^{k}, p-$ простое число. Предположим, что $G$ действует на $\mathbb{R}^{d}$ ортогонально транзитивными перестановками векторов некоторого базиса. Пусть замкнутый конус $C$ с иентром в начале координат таков, что семейство $\{ \pm g(C)\}_{g \in G} \partial a$ ет разбиение $\mathbb{R}^{d}$, а его подсемейство $\{g(C)\}_{g \in G}$ имеет единственный общий луч.

Тогда для любой абсолютно непрерывной вероятностной меры $\mu$ на $\mathbb{R}^{d}$ найдется такое не меняющее ориентацию движение $\rho$, что для любого $g \in G$

$$
\mu(\rho(g(C)))=\mu(\rho(-g(C)))=\frac{1}{2 d} .
$$

В доказательстве этой теоремы используется тот же класс Эйлера, что и в доказательстве теоремы 52 .

Рассмотрим теперь вопрос о разрезании мер на части заданной величины. Начнем с задачи о разбиении одной гиперплоскостью.

ЗАДАчА 2. Для заданных абсолютно непрерьвных мер $\mu_{1}, \ldots, \mu_{d}$ в $\mathbb{R}^{d}$ и чисел $\left(\alpha_{1}, \ldots, \alpha_{d}\right) \in[0,1]^{d}$ найти полупространство $H \subset \mathbb{R}^{d}$ такое, что для всех $i \in[d]$ выполнено равенство

$$
\mu_{i}(H)=\alpha_{i}
$$

При $\alpha_{i}=1 / 2$ эта задача решается положительно с помощью теоремы о бутерброде. В работе [99] изучалась эта задача и ее обобщение, в котором рассматривается $d+1$ мера и вместо полупространства требуется найти шар с тем 
же свойством. По сути, задача для шара сводится к приведенной выше с помощью отображения $f: \mathbb{R}^{d} \rightarrow \mathbb{R}^{d+1}$, действующего по формуле

$$
\left(x_{1}, \ldots, x_{d}\right) \mapsto\left(x_{1}, \ldots, x_{d}, x_{1}^{2}+\cdots+x_{d}^{2}\right) .
$$

Рассмотрение мер, равномерно распределенных на концентрических шарах, показывает, что в общем случае (без ограничений на меры) задача 2 может иметь решение только при $\alpha_{i}=1 / 2$. Что касается ограничений на меры, то в работе [99] установлен следующий частный случай, в котором задача 2 допускает положительное решение.

ОПРЕДЕЛЕНИЕ 43. Связные множества $C_{1}, \ldots, C_{k}$ в $\mathbb{R}^{d}$ вполне отделимы, если любой набор представителей $x_{i} \in C_{i}$ является аффинно независимым. Очевидно, что при этом $k \leqslant d+1$.

Теорема 62 (Барани, Хубард, Херонимо, 2008). Если выпуклые оболочки носителей мер $\mu_{i}$ вполне отделимы, то задача 2 решается при любых $\left(\alpha_{1}, \ldots, \alpha_{d}\right) \in[0,1]^{d}$.

Аналогичное утверждение верно и для $d+1$ меры и отрезания части мер шаром (см. [99]). В работе автора [100] условие на вполне отделимость было ослаблено следующим образом.

ОПРЕДЕЛЕНИЕ 44. Пару из непрерывной вероятностной меры $\mu$ с компактным носителем и числа $\varepsilon \in[0,1 / 2)$ будем называть мерой $c$ допуском. Для краткости при рассмотрении нескольких мер $\mu_{i}$ допуск каждой будем обозначать $\varepsilon\left(\mu_{i}\right)$.

ОПРедЕЛЕНиЕ 45. Пусть в $\mathbb{R}^{d}$ дана мера с допуском $\mu$. Будем говорить, что гиперплоскость $h$ пересекает (с допуском) меру $\mu$, если $h$ делит $\mathbb{R}^{d}$ на два полупространства $H_{1}$ и $H_{2}$ и

$$
\mu\left(H_{1}\right), \mu\left(H_{2}\right) \geqslant \varepsilon(\mu) .
$$

Гиперплоскости в $\mathbb{R}^{d}$ параметризуются пространством канонического расслоения $\gamma_{d}^{1}$ над грассманианом $G_{d}^{1}=\mathbb{R P}^{d-1}$ (см. п. 5.2).

ОПредЕЛЕНиЕ 46. Рассмотрим семейство из $d$ мер с допуском $\left\{\mu_{i}\right\}_{i=1}^{d}$ в $\mathbb{R}^{d}$. Обозначим через $X \subseteq \gamma_{d}^{1}$ множество гиперплоскостей, пересекающих с допуском все эти меры, и рассмотрим естественную проекцию $p: X \rightarrow G_{d}^{1}$. Будем говорить, что семейство мер с допуском $\left\{\mu_{i}\right\}_{i=1}^{d}$ nлоское, если отображение $p$ пропускается через отображение универсального накрытия $\pi: S^{d-1} \rightarrow G_{d}^{1}$, т. е. $p=\pi \circ \tilde{p}$.

Теорема 63 (Карасёв, 2008). Рассмотрим плоское семейство мер с допуском $\left\{\mu_{i}\right\}_{i=1}^{d}$ в $\mathbb{R}^{d}$ и числа $\left\{\alpha_{i}\right\}$ такие, что либо $\alpha_{i}=\varepsilon\left(\mu_{i}\right)$, либо $\alpha_{i}=1-\varepsilon\left(\mu_{i}\right)$. $B$ таком случае существует полупространство $H \subset \mathbb{R}^{d}$ такое, что для всех $i \in[d]$ выполнено равенство

$$
\mu_{i}(H)=\alpha_{i}
$$


Для простых конических разбиений в [94] получен следующий результат о разбиении на части заданной величины.

Теорема 64 (Вречица, Живалевич, 2001). Если $C_{1}, \ldots, C_{d+1}$ - nростое коническое разбиение $\mathbb{R}^{d}$, то для всякой абсолютно непрерывной вероятностной меры $\mu$ на $\mathbb{R}^{d}$ и набора положительных вещественных чисел $\left\{\alpha_{i}\right\}_{i \in[d+1]}$ с единичной суммой найдется такой вектор $c \in \mathbb{R}^{d}$, что для любого $i \in[d+1]$

$$
\mu\left(C_{i}+c\right)=\alpha_{i}
$$

В работе автора [101] был доказан аналог этого результата для деления меры на количество частей, большее $d+1$.

Tеорема 65 (Карасёв, 2005). Пусть $P$ - многогранник в $\mathbb{R}^{d}, a\left\{F_{i}\right\}_{i \in[m]}-$ набор его гиперграней. Предположим, что абсолютно непрерывная вероятностная мера н сосредоточена на $P$.

Тогда для любого набора положительных чисел $\left\{\alpha_{i}\right\}_{i \in[m]}$ с единичной суммой найдется разбиение $P$ на выпуклые множества $\left\{A_{i}\right\}_{i \in[m]}$, для которого при любом $i \in[\mathrm{m}]$

$$
A_{i} \cap \mathrm{bd} P=F_{i}, \quad \mu\left(A_{i}\right)=\alpha_{i}
$$

(здесъ bd - гранииа $P$ ).

В [95] сформулирована следующая гипотеза.

ГиПотезА 9 (Алон, 1987). Для каждого натуралъного $n$ и вещественного $\alpha \in(0,1)$ существует натуральное число $N(\alpha, n)$, обладающее следующим свойством.

Если на отрезке I заданы абсолютно непрерывные вероятностные меры $\mu_{1}, \ldots, \mu_{n}$, то найдется подмножество $X \subseteq I$, являющееся оббединением не более чем $N(\alpha, n)$ отрезков, для которого при любом $i \in[n]$

$$
\mu_{i}(X)=\alpha
$$

Для рациональных $\alpha$ эта гипотеза следует из многократного применения теоремы 59, для иррациональных $\alpha$ она остается открытой.

4.9. Топологический подход к теоремам типа Хелли. Следуя обзоpy [102], приведем основные понятия, используемые при топологическом подходе к теоремам типа Хелли.

ОПределЕниЕ 47. Для семейства $\mathscr{F}$ подмножеств $\mathbb{R}^{d}$ нервом семейства $\mathscr{F}$ назовем симплициальный комплекс $N(\mathscr{F})$ на вершинах $\mathscr{F}$, симплексами которого являются наборы $\sigma \subseteq \mathscr{F}$, имеющие непустое пересечение.

Для упрощения рассуждений далее будем рассматривать только конечные семейства подмножеств и конечные симплициальные комплексы. Основной результат о связи нерва с семейством множеств выглядит следующим образом [103]. 
Теорема 66 (Лере, 1945). Предположим, что множества семейства Я્F или все открытые, или все замкнутые. Также предположим, что для всякого подсемейства $\mathscr{G} \subseteq \mathscr{F}$ пересечение $\bigcap \mathscr{G}$ либо пусто, либо гомотопически тривиально. Тогда $\mathscr{G}$ слабо гомотопически эквивалентно нерву $N(\mathscr{F})$.

ОПредЕЛЕниЕ 48. Семейства, для которых верна теорема Лере, назовем правильными.

Ясно, что семейства замкнутых выпуклых множеств удовлетворяют условиям теоремы Лере. В связи с этим возникает вопрос [104].

ЗАДАчА 3 (Вегнер, 1975). Как охарактеризовать те симплиииальные комплексы, которые являются нервами семейств замкнутых выпуклых множеств в $\mathbb{R}^{d}$ ?

Назовем такие комплексы $d$-реализуемыми. Из теоремы Лере легко выводится следствие.

ТеОрема 67. Если комплекс $K$ является d-реализуемым, то для любого множества его вершин $W$ индуиированный подкомплекс $K[W]$ имеет гомологическую размерность не более $d-1$, иначе говоря, $\widetilde{H}^{i}(K[W], \mathbb{Z})=0$ при $i \geqslant d$.

В связи с этой теоремой уместно сделать определение.

ОПРЕДЕЛЕНИЕ 49. Рассмотрим симплициальный комплекс $K$ и число $d \geqslant 1$. Если для любого множества его вершин $W$ индуцированный подкомплекс $K[W]$ имеет гомологическую размерность не более $d-1$, то комплекс $K$ назовем $d$-мерным по Лере комплексом. Общепринятый англоязычный термин " $d$-Leray complex".

В работе [105] был приведен пример 2-мерного по Лере комплекса, который не является 2-реализуемым. В связи с этим имеет смысл искать более точные характеризации $d$-реализуемых комплексов.

ОПРЕДЕЛЕНИЕ 50. Элементарным $d$-складыванием комплекса назовем удаление некоторого симплекса $\sigma$, размерности не более $d-1$, который содержится в единственном максимальном симплексе, вместе с $\sigma$ удаляются все содержащие его симплексы. Комплекс называется $d$-складным, если последовательностью элементарных $d$-складываний можно превратить комплекс в пустой. Общепринятые англоязычные термины "elementary $d$-collapse" и " $d$-collapsible".

Теорема 68 (Вегнер [104], 1975). Всякий d-реализуемый комплекс является d-складным.

Свойство $d$-складности влечет справедливость теоремы Хелли, цветной теоремы Хелли, дробной теоремы Хелли (см. ниже), теоремы Хадвигера-Дебруннера (см. ниже) и т. п.

ДОКАЗАТЕЛЬСТВо ТЕОРЕМЫ 68. Без ограничения общности можно считать, что выпуклые множества в семействе $\mathscr{F}$ являются строго выпуклыми компактами, причем их границы являются гладкими поверхностями, находящимися 
в общем положении в $\mathbb{R}^{d}$. В частности, это означает, что никакие $d+1$ границы не пересекаются в одной точке. Если в семействе не более $d$ элементов, то нерв $d$-складной по определению.

Рассмотрим теперь некоторую линейную функцию $l: \mathbb{R}^{d} \rightarrow \mathbb{R}$ (выбор функции будет пояснен ниже) и положим

$$
l_{0}=\max _{\mathscr{G} \subseteq \mathscr{F}, \bigcap \mathscr{G} \neq \varnothing} \min _{x \in \cap \mathscr{G}} l(x) .
$$

Пусть значение $l_{0}$ достигается для некоторого $\mathscr{G}$ в точке $x_{0}$. Из общего положения ясно, что $x_{0}$ лежит на границах не более $d$ множеств, тогда можно перейти к подсемейству и считать, что $|\mathscr{G}| \leqslant d$ и для любого $C \in \mathscr{G} x_{0} \in \partial C$.

Подсемейство $\mathscr{G}$ с точкой $x_{0}$ при заданном уровне $l_{0}$ вообще может выбираться неоднозначно. Но границы множеств $\mathscr{F}$ находятся в общем положении, поэтому можно изначально выбрать такую функцию $l$, что подсемейство $\mathscr{G}$ будет выбираться однозначно.

Легко видеть, что если для некоторого $C \in \mathscr{F} \backslash \mathscr{G}$ пересечение $C \cap \bigcap \mathscr{G}$ непусто, то $C \ni x_{0}$. Следовательно, грань $\mathscr{G}$ нерва $N(\mathscr{F})$ содержит единственную максимальную грань, соответствующую тем множествам, которые содержат $x_{0}$.

Легко видеть, что если взять достаточно малое $\varepsilon$ и рассмотреть полупространство $H=\left\{x \in \mathbb{R}^{d}: l(x) \leqslant l_{0}-\varepsilon\right\}$, то замена семейства $\mathscr{F}$ на семейство

$$
\mathscr{F}^{\prime}=\{F \cap H: F \in \mathscr{F}\}
$$

приводит именно к складыванию симплекса $\mathscr{G}$. Далее процесс повторяется. Теорема доказана.

Известно, что уже при $d=2$ можно привести пример $d$-мерного по Лере комплекса, который не является $d$-складным. Кроме того, в обзоре [102] вводится понятие строгой $d$-складности, которое также следует из $d$-реализуемости.

Приведем некоторые теоремы типа Хелли для семейств выпуклых множеств.

Теорема 69 (дробная теорема Хелли; Качальски, Лю [106], 1979). Для всякого $\beta>0$ найдется такое $\alpha>0$, что если в конечном семействе выпуклых компактов $\mathscr{F}$ в $\mathbb{R}^{d}$ c $|\mathscr{F}|=n$ найдется не менее $\beta\left(\begin{array}{c}n \\ d+1\end{array}\right)$ подсемейств из $d+1$ множества с общей точкой, то найдется подсемейство $\mathscr{G} \subseteq \mathscr{F}$ такое, что

$$
|\mathscr{G}| \geqslant \alpha n, \quad \bigcap \mathscr{G} \neq \varnothing .
$$

В работе [106] также приведены явные формулы для некоторой конкретной функции $\alpha(\beta, d)$.

ОПРЕДЕЛЕНИЕ 51. Будем говорить, что семейство множеств $\mathscr{F}$ имеет точечную $n$-трансверсаль, если существует $n$-элементное множество $T$, пересекающее все множества семейства $\mathscr{F}$. Минимальный размер трансверсали $\mathscr{F}$ обозначается $\tau(\mathscr{F})$, его англоязычное название "piercing number".

ОПРЕДЕЛЕНИЕ 52. Будем говорить, что для семейства множеств $\mathscr{F}$ выполняется $(p, q)$-свойство, если $|\mathscr{F}| \geqslant p$ и в любом подсемействе $\mathscr{G}$ из $p$ элементов найдется подсемейство $\mathscr{H}$ из $q$ элементов, для которого $\bigcap \mathscr{H} \neq \varnothing$. 
Теорема 70 (Хадвигер, Дебруннер [107], 1957). Предположим, что $p \geqslant q \geqslant$ $d+1$ и $p(d-1)<(q-1) d$. Тогда для всякого семейства выпуклых множеств Я в $\mathbb{R}^{d}$ из $(p, q)$-свойства следует $\tau(\mathscr{F}) \leqslant p-q+1$.

Теорема 71 (Алон, Клейтман [108], 1992). Предположим, что $p \geqslant q \geqslant d+1$. Тогда найдется такое число $t(p, q, d)$, что для всякого семейства выпуклых множеств $\mathscr{F}$ в $\mathbb{R}^{d}$ из $(p, q)$-свойства следует $\tau(\mathscr{F}) \leqslant c(p, q, d)$.

Очевидно, что в теореме Хелли достаточно выполнения свойства $d$-мерности по Лере для нерва семейства множеств. Поэтому возникает естественный вопрос: насколько в теоремах типа Хелли требуется $d$-реализуемость нерва, $d$-складность нерва или достаточно $d$-мерности по Лере? Приведем некоторые результаты в этом направлении.

В работе [109] показано, что для выполнения дробной теоремы Хелли и теоремы 71 достаточно выполнения свойства $d$-мерности по Лере для нерва семейства множеств. В работе [110] показано, что для выполнения цветной теоремы Хелли достаточно того же свойства. То есть фактически указанные теоремы выполняются для любого правильного семейства множеств в $\mathbb{R}^{d}$.

4.10. Теоремы типа Кнастера-Куратовского-Мазуркевича. Сформулируем обобщение теоремы KKM о покрытиях симплекса, которое можно рассматривать как цветной вариант теоремы KKM, аналогично цветному варианту теоремы Хелли. Частный случай $(m=n, a(x) \equiv 1)$ доказан в работе [111], приведенная здесь формулировка доказана автором в [112].

Tеорема 72 (Бапат, Карасёв, 1989-2006). Рассмотрим множество $X$ из $n$ точек в $\mathbb{R}^{d}$ и множество индексов $[m]$, где $m \geqslant n$. Пусть $A(x, j)$, где $x \in X$, $j \in[m],-$ семейство замкнутых множеств в $\mathbb{R}^{d}$, для которого

$$
\forall j \in[m] \quad \forall Y \subseteq X \quad \operatorname{conv} Y \subseteq \bigcup_{x \in Y} A(x, j) .
$$

Пусть каждому $x \in X$ сопоставлено натуральное число а $(x)$ так, что

$$
\sum_{x \in X} a(x)=m
$$

Тогда найдется такое отображение множества индексов $\sigma:[m] \rightarrow X$, что

$$
\forall x \in X \quad\left|\sigma^{-1}(x)\right|=a(x), \quad \bigcap_{j=1}^{m} A(\sigma(j), j) \neq \varnothing .
$$

Обобщением этой теоремы является аналог теоремы KКМ для произведения симплексов, доказанный в [113] (см. также [114]) для случая $m=n$, $a_{i} \equiv 1$, который мы приводимый здесь в более общей формулировке, полученной автором.

Теорема 73 (Тардош, Карасёв, 1995-2008). Предположим, что произведение симплексов $\Delta^{n-1} \times \Delta^{m-1}(m \geqslant n)$ покрыто семейством из тn замкнутых множеств $A_{i j}(i \in[n], j \in[m])$, причем каждое $A_{i j}$ не пересекает ни $\partial_{i} \Delta^{n-1} \times \Delta^{m-1}$, ни $\Delta^{n-1} \times \partial_{j} \Delta^{m-1}$. Возъмем натуральнье числа $a_{1}, \ldots, a_{n}$, сумма которьх равна $m$. 
Тогда найдется отображение $\sigma:[m] \rightarrow[n]$, для которого

$$
\forall i \in[n] \quad\left|\sigma^{-1}(i)\right|=a_{i}, \quad \bigcap_{j=1}^{m} A_{\sigma(j) j} \neq \varnothing .
$$

Приведем доказательство этой теоремы. Сначала сформулируем лемму, которая легко доказывается индукцией по остовам комплекса.

Лемма 5. Пусть непрерывное отображение $f: \Delta^{d} \rightarrow \Delta^{d}$ отображает каждую грань $\Delta^{d}$ в себя. Тогда отображение $f^{*}: H^{d}\left(\Delta^{d}, \partial \Delta^{d}\right) \rightarrow H^{d}\left(\Delta^{d}, \partial \Delta^{d}\right)$ тождественно и само отображение $f$ сюргективно.

ДОКАЗАТЕЛЬСтво теОРемы 73. Сначала от множеств $A_{i j}$ перейдем к функциям $\chi_{i j}: \Delta^{n-1} \times \Delta^{m-1} \rightarrow \mathbb{R}$, которые равны единице на $A_{i j}$ и обращаются в нуль в некоторой $\varepsilon$-окрестности $A_{i j}$.

Так как множества $A_{i j}$ покрывают $\Delta^{n-1} \times \Delta^{m-1}$, то можно перейти к нормированным функциям

$$
\phi_{i j}(x)=\frac{\chi_{i j}(x)}{\sum_{k, l} \chi_{k l}(x)} .
$$

Далее рассмотрим функции

$$
f_{i}(x)=\sum_{j=1}^{m} \phi_{i j}(x), \quad g_{j}(x)=\sum_{i=1}^{n} \phi_{i j}(x) .
$$

Эти наборы функций дают отображения $f: \Delta^{n-1} \rightarrow \Delta^{n-1}$ (при фиксированном вложении $\Delta^{n-1} \times\{y\} \rightarrow \Delta^{n-1} \times \Delta^{m-1}$ ) и $g: \Delta^{m-1} \rightarrow \Delta^{m-1}$ (при фиксированном вложении $\left.\{x\} \times \Delta^{m-1} \rightarrow \Delta^{n-1} \times \Delta^{m-1}\right)$, при достаточно малом $\varepsilon$ удовлетворяющие условиям леммы 5 . Тогда по лемме 5 отображения

$$
f^{*}: H^{*}\left(\Delta^{n-1}, \partial \Delta^{n-1}\right) \rightarrow H^{*}\left(\Delta^{n-1} \times \Delta^{m-1}, \partial \Delta^{n-1} \times \Delta^{m-1}\right)
$$

и

$$
g^{*}: H^{*}\left(\Delta^{m-1}, \partial \Delta^{m-1}\right) \rightarrow H^{*}\left(\Delta^{n-1} \times \Delta^{m-1}, \Delta^{n-1} \times \partial \Delta^{m-1}\right)
$$

тождественны. По свойству $\times$-произведения когомологий отображение $(f \times g)$ * тождественно на $H^{n+m-2}\left(\Delta^{n-1} \times \Delta^{m-1}, \partial\left(\Delta^{n-1} \times \Delta^{m-1}\right)\right)$.

Значит, $f \times g$ сюръективно и найдется точка $x$, для которой

$$
\forall i \in[n] \quad f_{i}(x)=\frac{a_{i}}{m}, \quad \forall j \in[m] \quad g_{j}(x)=\frac{1}{m} .
$$

Возвращаясь к матрице $\phi_{i j}(x)$, видим, что у нее суммы по строкам равны $a_{i} / m$, а по столбцам $1 / m$. Значит, по лемме Холла о паросочетаниях найдется отображение $\sigma:[m] \rightarrow[n]$, для которого

$$
\forall i \in[n] \quad\left|\sigma^{-1}(i)\right|=a_{i}, \quad \forall j \in[m] \quad \phi_{\sigma(j) j}(x) \neq 0 .
$$

Далее утверждение теоремы получается переходом к пределу $\varepsilon \rightarrow 0$. Теорема доказана. 


\section{5. Плоские трансверсали и топология многообразий Грассмана}

5.1. Теоремы типа Хелли для плоских трансверсалей. В комбинаторной геометрии теоремами типа Хелли называются утверждения, в которых для проверки некоторого свойства семейства множеств нужно проверить это (или иное) свойство в каждом подсемействе ограниченного сверху некоторой константой размера. В самой теореме Хелли свойство иметь общую точку нужно проверить для всех подсемейств размера не более $d+1$.

ОПРедЕЛЕНиЕ 53. Пусть $\mathscr{F}$ - семейство подмножеств $\mathbb{R}^{d} \cdot k$-плоскость $L$ называется плоской $k$-трансверсалью семейства $\mathscr{F}$, если для любого $C \in \mathscr{F}$ пересечение $C \cap L$ непусто. Англоязычный термин "common transversal".

Далее плоские трансверсали будем называть просто трансверсалями, если это не вызывает недоразумений. Уже в случае $k=1$ (линейные трансверсали) на плоскости не существует аналога теоремы Хелли для проверки существования трансверсали.

ПримеР 1. Впишем в круг радиуса 1 правильный $2 n$-угольник с вершинами $\left\{p_{i}\right\}_{i \in[2 n]}$. Рассмотрим семейство равных кругов $B_{i}$ с радиусами $\frac{1}{2}\left(1+\cos \frac{\pi}{n}\right)$ с центрами в точках $p_{i}$. Легко проверить, что любые $2 n-1$ круга семейства можно пересечь прямой, но все пересечь нельзя. Таким образом, для наличия линейной трансверсали на плоскости константы Хелли не существует.

Тем не менее, на плоскости есть некоторые положительные результаты в этом направлении.

Теорема 74 (Хадвигер [115], 1957). Рассмотрим семейство Я્F из не менее чем трех попарно непересекаюшихся выпукльх компактов в $\mathbb{R}^{2}$. Предположим, что $\mathscr{F}$ можно линейно упорядочить так, что всякие три множества можно пересечь прямой в порядке, совпадающем с введенным порядком на Ғ․ Тогда Я્F имеет линейную трансверсаль.

ТеОРема 75 (Качальски, Льюис [116], 1980). Существует такая константа $k$, что выполняется следующее условие. Пусть $\mathscr{F}$ - семейство попарно непересекающихся транслятов одного и того же выпуклого компакта $C \in \mathbb{R}^{2}$, в котором каждое подсемейство, состоящее из трех или менее множеств, имеет линейную трансверсаль. Тогда из Я્F можно выбросить не более $k$ множеств так, что оставшиеся будут иметь линейную трансверсаль.

Теорема 76 (Тверберг [117], 1989). Семейство $\mathscr{F}$ из не менее пяти попарно непересекающихся транслятов одного и того же выпуклого компакта $C \in \mathbb{R}^{2}$ имеет линейную трансверсаль, если линейную трансверсаль имеет каждое подсемейство $\mathscr{G} \subseteq \mathscr{F}$, для которого $|\mathscr{G}|=5$.

Кроме того, получены некоторые результаты для линейных трансверсалей семейств равных шаров в $\mathbb{R}^{d}$. Один из них приведен ниже.

Теорема 77 (Холмсен, Качальски, Льюис, Чон, Гоаок, Птижан [118], [119], 2003-2008). Семейство $\mathscr{F}$ из не менее 4d-1 попарно непересекающихся 
единичных шаров в $\mathbb{R}^{d}$ имеет линейную трансверсаль, если линейную трансверсаль имеет каждое подсемейство $\mathscr{G} \subseteq \mathscr{F}$, для которого $|\mathscr{G}|=4 d-1$.

В работе [120] показано, что не существует теоремы типа Хелли, позволяющей доказывать существование линейной трансверсали семейства попарно непересекающихся транслятов одного и того же компакта в $\mathbb{R}^{3}$, что опровергает гипотезу из работы [121]. Для трансверсалей большей размерности ситуация еще сложнее. Во-первых, ясно, что для существования теоремы типа Хелли для $k$-плоскостей надо потребовать выполнения некоторого свойства, аналогичного отсутствию попарных пересечений в случае линейных трансверсалей.

ОПРеДЕЛЕНиЕ 54. Семейство выпуклых компактов в $\mathbb{R}^{d}$ назовем $k$-отделимым, если любое его подсемейство из не более чем $k+2$ множеств вполне отделимо (см. определение 43).

В соответствии с этим определением, 0-отделимость - это отсутствие попарных пересечений. Нетрудно заметить, что для установления теоремы типа Хелли для $k$-трансверсалей необходимо требовать $(k-1)$-отделимость. В работе [122] доказано следующее обобщение теоремы Хадвигера о линейной трансверсали.

Теорема 78 (Гудман, Поллак, 1988). Предположим, что для $(d-2)$-отделимого семейства $\mathscr{F}$ выпуклых компактов в $\mathbb{R}^{d}$ найдется ориентированный матроид ранга d на множестве $\mathscr{F}$, для которого выполняется следующее свойство. Любое подсемейство $\mathscr{G} \subseteq \mathscr{F}$ из $d+1$ множеств имеет гиперплоскую трансверсаль $h$, и пересечения $\{C \cap h\}_{C \in \mathscr{G}}$ расположены в $h$ согласованно с матроидом. Тогда $\mathscr{F}$ имеет гиперплоскую трансверсаль.

В работе [121] с помощью предыдущей теоремы был установлен следующий результат.

Теорема 79 (Аронов, Гудман, Поллак, Венгер, 2000). Для любого $d u \varepsilon>0$ существует число $N(d, \varepsilon)$, обладающее следующим свойством. Предположим, что семейство $\mathscr{F}$ из не менее $N(d, \varepsilon)$ выпуклых компактов диаметра $\leqslant 1$ в $\mathbb{R}^{d}$ таково, что семейство в-окрестностей множеств из $\mathscr{F}$ является $(d-2)$-отделимым. Тогда для наличия гиперплоской трансверсали Ғ્F достаточно проверить наличие гиперплоской трансверсали всех подсемейств $\mathscr{G} \subseteq \mathscr{F}$ размера не более $2 d-2$.

Там же была сформулирована гипотеза, что просто 1-отделимость достаточна для существования теоремы типа Хелли для 2-трансверсалей семейств равных шаров в $\mathbb{R}^{3}$. Однако в работе [123] был построен контрпример к этой гипотезе.

Приведенные выше результаты показывают, что вопрос о существовании теорем типа Хелли для плоских трансверсалей сам по себе довольно сложен. Часто можно получить результат о $k$-трансверсалях с помощью ортогональной проекции на дополнительную $(d-k)$-плоскость. Типичный пример дает теорема Хорна и Кли [124], [125]. 
Теорема 80 (Хорн, Кли, 1949-1951). Для натуральных $1 \leqslant k \leqslant d$ и семейства $\mathscr{F}$ выпуклых компактов в $\mathbb{R}^{d}$ следующие три условия эквивалентны:

1) каждые $\leqslant k$ множеств из $\mathscr{F}$ имеют общую точку;

2) каждая плоскость коразмерности $k-1$ в $\mathbb{R}^{d}$ имеет транслят, пересекающий все множества $\mathscr{F}$;

3) каждая плоскость коразмерности $k$ в $\mathbb{R}^{d}$ принадлежит плоскости коразмерности $k-1$, пересекающей все множества $\mathscr{F}$.

\section{2. Топология канонического расслоения над многообразием}

Грассмана. Для применения топологических методов к задачам о плоских трансверсалях нужно подходящим образом параметризовать пространство всех $k$-плоскостей.

Рассмотрим пространство всех $k$-плоскостей в $\mathbb{R}^{d}$. Каждой такой плоскости $\alpha$ можно поставить в соответствие единственное ортогональное ей $(d-k)$ мерное линейное подпространство $g(\alpha) \subset \mathbb{R}^{d}$ и единственную точку пересечения $\alpha \cap g(\alpha)$. Таким образом, пространство $k$-мерных аффинных плоскостей отождествляется с пространством $\gamma_{d}^{d-k}$ канонического расслоения над вещественным грассманианом $G_{d}^{d-k}$. В дальнейшем будем считать пространство плоскостей наделенным топологией $\gamma_{d}^{d-k}$.

Если нам нужно рассмотреть пространство ориентированных $k$-плоскостей, то оно естественным образом отождествляется с пространством $\gamma_{d}^{d-1^{+}}$канонического расслоения над ориентированным грассманианом $G_{d}^{d-k^{+}}$.

Следующая теорема о классе Эйлера канонического расслоения для $p=2$ доказана в [126], случай одновременно нечетных $p$ и $d$ доказан в [127], приведенная здесь формулировка получена автором в [128].

Теорема 81 (Дольников, Живалевич, Карасёв, 1987-2007). Пусть p - простое число. Тогда, если $p=2$, то

$$
e\left(\gamma_{d}^{d-k}\right)^{k} \neq 0 \in H^{d(d-k)}\left(G_{d}^{d-k}, Z_{p}\right)
$$

а если $p>2$ ud-k-четно, то

$$
e\left(\gamma_{d}^{d-k^{+}}\right)^{k} \neq 0 \in H^{d(d-k)}\left(G_{d}^{d-k^{+}}, Z_{p}\right)
$$

В векторном расслоении $\gamma_{d}^{d-k} \rightarrow G_{d}^{d-k}$ циклическая группа $Z_{2}$ действует отображением $x \mapsto-x$ в каждом слое. В некоторых приложениях необходимо знать $Z_{2}$-индекс пространства сфер $S\left(\gamma_{d}^{d-k}\right)$ этого расслоения [100].

Tеорема 82 (Карасёв, 2008). ind $\underline{Z}_{2} S\left(\gamma_{d}^{d-k}\right)=d-1$.

Рассмотрим теперь ориентированный грассманиан $G_{d}^{k^{+}}$. На нем $Z_{2}$ действует сменой ориентации. Заметим, что взятие ортогонального дополнения дает канонический изоморфизм $G_{d}^{k^{+}} \sim G_{d}^{d-k^{+}}$, перестановочный с операцией замены ориентации. Поэтому для вычисления $Z_{2}$-индекса ориентированного грассманиана достаточно рассмотреть случай $2 k \leqslant d$. Следующая теорема суммирует сведения об индексе ориентированного грассманиана из работ [129], [130]. 
Теорема 83 (Хиллер, 1980). Пусть $2 k \leqslant d u 2^{s}$ - минимальное целое число maкое, что $2^{s} \geqslant d$.

1) Eсли $k=1$, mo $\underline{\text { ind }}_{Z_{2}} G_{d}^{1^{+}}=\underline{\text { ind }}_{Z_{2}} S^{d-1}=d-1$.

2) $E$ сли $k=2$, mo ind ${ }_{Z_{2}} G_{d}^{k^{+}}=2^{s}-2$.

3) Если $k>2$, то при $d=2 k=2^{s} 2^{s-1} \leqslant \underline{\operatorname{ind}}_{Z_{2}} G_{d}^{k^{+}} \leqslant 2^{s}-1$, в остальньх случаях $2^{s}-2 \leqslant \underline{\text { ind }}_{z_{2}} G_{d}^{k^{+}} \leqslant 2^{s}-1$.

Во всех случаях ⿱ㅐㅇ $Z_{2} G_{d}^{k^{+}} \geqslant d-k$ и равенство достигается, только если $k=1$ или одновременно $k=2$ ud $=2^{s}$.

Следующее утверждение о грассманиане применяется в статье [131].

Теорема 84 (Громов, 1967). Рассмотрим вещественный (или комплексный) грассманиан $G_{d}^{k}$ и каноническое векторное расслоение $\gamma_{d}^{k} \rightarrow G_{d}^{k}$. Тогда структурная группа этого расслоения не может быть редуцирована к собственной подгруппе $O(k)$ (в комплексном случае $U(k)$ ), если выполняется одно из следующих условий:

1) $d \geqslant 2 k$ для комплексного грассманиана;

2) $k$ нечетно $и d \geqslant 3 k-2$ для вещественного грассманиана;

3) $k$ четно и $d \geqslant 3 k-4$ для вещественного грассманиана.

Кроме того, структурная группа расслоения не редуцируется $к$ подгруппе, действующей нетранзитивно на $(k-1)$-мерной сфере $((2 k-1)$-мерной сфере в комплексном случае) при четных $k$.

С помощью этого утверждения в работе [131] доказаны некоторые частные случаи гипотезы Банаха.

ГиПотезА 10 (Банах). Пусть $1<k<n$. Предположим, что у $n$-мерного банахова пространства $B$ (вещественного или комплексного) все $k$-мерные подпространства изоморфны как банаховы пространства. Тогда пространство В евклидово.

\section{3. Теорема Дольникова и другие теоремы типа Хелли о плоских} трансверсалях. Важным следствием теоремы 81 является следующая теорема из [126].

ОПРеДЕЛЕНИЕ 55. Множество $X \subseteq \mathbb{R}^{n}$ называется $l$-выпуклъм, если его проекция на любое $l$-мерное подпространство $\mathbb{R}^{n}$ выпукла.

Теорема 85 (Дольников, 1987). Рассмотрим в $\mathbb{R}^{d}$ семейства $\mathscr{F}_{0}, \ldots, \mathscr{F}_{k}$ $(d-k)$-выпуклых компактов. Предположим, что в каждом семействе любые $d-k+1$ или менее множеств имеют общую точку. Тогда семейство $\bigcup_{i=0}^{k} \mathscr{F}_{i}$ имеет k-трансверсаль.

В работе [132] приводится еще одно следствие теоремы 81.

ОПРЕДЕЛЕНИЕ 56 . Назовем $k$-плоскость $L$ максималъной $k$-трансверсалъю выпуклого компакта $K$, если

$$
\operatorname{mes}_{k} L \cap K=\max _{L^{\prime} \| L} \operatorname{mes}_{k} L^{\prime} \cap K .
$$


Здесь $\operatorname{mes}_{k}$ - нормализованная мера на $k$-мерной плоскости $L$ или $L^{\prime}$. Максимальная 1-трансверсаль называется аффинным диаметром.

Теорема 86 (Макаи, Вречица, Живалевич, 2001). Для всякого семейства $K_{0}, \ldots, K_{k}$ из $k+1$ выпуклого компакта в $\mathbb{R}^{d}$ найдется $k$-плоскость $L$, которая является максимальной трансверсалью одновременно для всех $K_{i}$.

В работе [132] приводятся и другие следствия теоремы 81, в частности, характеризующие множество общих максимальных $k$-трансверсалей для семейства из $l \leqslant k$ выпуклых компактов.

Ниже приводятся некоторые результаты автора из [100], которые являются теоремами типа Хелли для плоских трансверсалей. Следующая теорема близка к теореме Дольникова и является следствием теоремы типа Борсука-УламаЛюстерника-Шнирельмана для сферы.

Теорема 87 (Карасёв, 2008). Пусть в $\mathbb{R}^{d}$ дано $d+1$ семейство 1-выпукльх компактов $\left\{\mathscr{F}_{i}\right\}_{i \in[d+1]}$. Пусть в каждом семействе любые два компакта пересекаются.

Тогда выполняется одна из следующих альтернатив:

1) семейство $\bigcup_{i \in[d+1]} \mathscr{F}_{i}$ имеет $(d-1)$-трансверсаль;

2) для всякого разбиения множества индексов $[d+1]$ на два множества $I_{1}$ и $I_{2}$ найдутся такие гиперплоскость $h$ и набор представителей $C_{i} \in \mathscr{F}_{i}$ $(i \in[d+1])$, что множества $\left\{C_{i}\right\}_{i \in I_{1}}$ лежат с одной стороны от гиперплоскости $h, a\left\{C_{i}\right\}_{i \in I_{2}}$ лежат с другой стороны от нее.

Следующая теорема является следствием теоремы 83.

Теорема 88 (Карасёв, 2008). Пусть $0<k<d$ и в $\mathbb{R}^{d}$ дано $d-k+1$ семейство $\left\{\mathscr{F}_{i}\right\}_{i \in[d-k+1]}$ выпуклых компактов. Тогда выполняется одна из следующих альтернатив:

1) найдется система представителей $K_{i} \in \mathscr{F}_{i}$ такая, что

$$
\bigcap_{i \in[d-k+1]} K_{i}=\varnothing
$$

2) в некотором из семейств $\mathscr{F}_{i}$ любие $k+1$ или менее множеств имеют $(k-1)$-трансверсаль;

3) найдется семейство параллельных $k$-плоскостей $\left\{\alpha_{i}\right\}_{i \in[d-k+1]}$ такое, что для всех $i \in[d-k+1] k$-плоскость $\alpha_{i}$ является $k$-трансверсалью семейства $\mathscr{F}_{i}$.

При этом третъя альтернатива возможна, только если $2 k \leqslant d, k=1$ uли $k=2$ u $d=2^{l}$.

В случае, когда количество семейств невелико по сравнению с $d$, утверждение теоремы 88 можно уточнить.

Tеорема 89 (Карасёв, 2008). Пусть $d=2 k+1 \geqslant 3$ и в $\mathbb{R}^{d}$ данъь два семейства $\mathscr{F}_{1}, \mathscr{F}_{2}$ выпуклых компактов. Тогда выполняется одна из следующих альтернатив: 
1) найдутся два представителя $K_{1} \in \mathscr{F}_{1}, K_{2} \in \mathscr{F}_{2}$, для которых $K_{1} \cap$ $K_{2}=\varnothing$;

2) в некотором из семейств $\mathscr{F}_{i}$ любие $k+2$ или менее множеств имеют $k$-трансверсаль.

Следующая теорема является аналогом теоремы 88, полученным с помощью теоремы 83 об индексе ориентированного грассманиана.

Tеорема 90 (Карасёв, 2008). Пусть $k>2,2 k<d+2$ и в $\mathbb{R}^{d}$ дано $k$ ceмейств $\mathscr{F}_{1}, \ldots, \mathscr{F}_{k}$ выпукльх компактов. Пусть для некоторого $m \leqslant d-k+1$ выполнено неравенство

$$
2^{\left\lceil\log _{2} d\right\rceil} \geqslant k \cdot 2^{\left\lceil\log _{2}(d-m)\right\rceil}+2 .
$$

Тогда выполняется одна из следующих альтернатив:

1) найдется система представителей $K_{1} \in \mathscr{F}_{1}, \ldots, K_{k} \in \mathscr{F}_{k}$, для которых $\bigcap_{i=1}^{k} K_{i}=\varnothing$

2) в некотором из семейств $\mathscr{F}_{i}$ любые $m+1$ или менее множеств имеют $(m-1)$-трансверсаль.

\section{4. Теорема об общей неподвижной точке послойных отображе-} ний. Сформулируем обобщение теоремы Брауэра о неподвижной точке из работы автора [68], в котором фигурируют несколько послойных отображений пространства шаров векторного расслоения.

Теорема 91 (Карасёв, 2008). Пусть дано векторное расслоение $\pi_{V}: V \rightarrow X$ u число $k \geqslant 0$, причем для класса Эйлера выполняется $е(V)^{k} \neq 0$. Пусть дано $k+1$ послойное непрерывное отображение пространства шаров $f_{i}$ : $B(V) \rightarrow B(V)(i=1, \ldots, k+1)$. Тогда найдется точка $x \in B(V)$, для которой

$$
x=f_{1}(x)=\cdots=f_{k+1}(x) .
$$

5.5. Теоремы о центральной точке и Тверберга для трансверсалей. В работах [133]-[135] доказана следующая теорема о трансверсалях, обобщающая как теорему о центральной точке $(k=0)$, так и теорему о бутерброде $(k=d-1)$.

Теорема 92 (Живалевич, Вречица, Дольников, 1990-1992). Пусть на $\mathbb{R}^{d}$ задана $k+1$ абсолютно непрерьвная вероятностная мера $\mu_{0}, \ldots, \mu_{k}$. Тогда найдется $k$-плоскость $L \in \mathbb{R}^{d}$ такая, что для всякого полупространства $H \supseteq L$ и всякого $i=0, \ldots, k$

$$
\mu_{i}(H) \geqslant \frac{1}{d-k+1}
$$

В работе автора [68] с помощью теоремы 91 был доказан двойственный вариант этой теоремы (см. обозначение $I(M, k)$ в п. 4.5 , определение 31$)$.

Теорема 93 (Карасёв, 2008). Пусть на множестве $\gamma_{d}^{d-k}$ заданы $d-k$ абсолютно непрерывных вероятностных мер $\mu_{1}, \ldots, \mu_{d-k}$ с компактными носителями. Тогда найдется $(d-k-1)$-плоскость $L$ такая, что для всякой 
$(d-k)$-полуплоскости $M$ с краем $L$ и любого $i=1, \ldots, d-k$

$$
\mu_{i}(I(M, k)) \geqslant \frac{1}{k+2} .
$$

В 1989 г. на Симпозиуме по комбинаторике и геометрии в Стокгольме Тверберг сформулировал следующую гипотезу, которая обобщает теорему 92 в том же духе, как теорема Тверберга обобщает теорему о центральной точке.

ГиПотезА 11. Возъмем число $0 \leqslant k \leqslant d-1$ и конечные множества $S_{0}$, $S_{1}, \ldots, S_{k}$ в $\mathbb{R}^{d}$. Предположим, что $\left|S_{i}\right|=\left(r_{i}-1\right)(d-k+1)+1$. Тогда каждое из множеств $S_{i}$ можно разбить на $r_{i}$ частей $S_{i 1}, S_{i 2}, \ldots, S_{i r_{i}}$ так, что множества conv $S_{i j}\left(i=0, \ldots, k, j \in\left[r_{i}\right]\right)$ будут иметь $k$-трансверсаль.

В работах [136], [127], [137] были доказаны некоторые частные случаи этой гипотезы, в которых требовалась простота чисел $r_{i}$. На самом деле доказательства носили топологический характер и формулировались для отображений симплексов в $\mathbb{R}^{d}$. В работе автора [128] была получена более общая формулиpoвка.

Теорема 94 (Тверберг, Вречица, Живалевич, Карасёв, 1993-2007). Возъмем число $0 \leqslant m \leqslant d-1$, пусть $r_{i}(i=0,1, \ldots, m)$ - степени одного и того же простого числа $r_{i}=p^{k_{i}}$. Для нечетных $p$ пусть $d-m$ четно.

Пусть для всех $i=0,1, \ldots, m$ отображение $f_{i}$ непрерывно отображает $\left(r_{i}-1\right)(d-m+1)$-мерный симплекс $\Delta_{i}=\Delta^{\left(r_{i}-1\right)(d-m+1)}$ в $\mathbb{R}^{d}$.

Тогда в каждом $\Delta_{i}$ найдутся $r_{i}$ попарно непересекающихся граней $F_{i 1}$, $F_{i 2}, \ldots, F_{\text {ir }_{i}} \in \Delta_{i}$ такие, что семейство $\left\{f_{i}\left(F_{i j}\right)\right\}_{0 \leqslant i \leqslant m, j \in\left[r_{i}\right]}$ имеет т-трансверсаль.

Также в работе [128] был доказан аналог топологической цветной теоремы Тверберга для трансверсалей. Его формулировка отличается от приведенного выше утверждения тем, что вместо симплексов $\Delta_{i}$ рассматриваются $(d+1)$-кратные джойны $K_{i}=\left[t_{i}\right]^{* d+1}$, где $t_{i}=2 r_{i}-1$.

5.6. Теоремы типа Борсука-Улама для трансверсалей. В работе автора [100] из теоремы 82 выводятся некоторые следствия типа Борсука-Улама для плоских трансверсалей.

ОПРедЕЛЕНИЕ 57. Пару точек на границе выпуклого компакта $K \subset \mathbb{R}^{d}$ назовем антиподальной по отношению к $K$, если их можно заключить в пару опорных гиперплоскостей к $K$ с противоположными нормалями.

ОПредЕление 58. Семейство компактов $\mathscr{F}$ в $\mathbb{R}^{d}$ назовем неантиподальнъцм, если никакое $V \in \mathscr{F}$ не содержит пары антиподальных точек по отношению к conv $\mathscr{F}$.

ТеОрема 95 (Карасёв, 2008). Если в $\mathbb{R}^{d}$ дано неантиподальное семейство компактов $\mathscr{F},|\mathscr{F}|=d+1$, то найдется $k$-плоскость, находящаяся от всех множеств семейства на равном расстоянии. Если, кроме того, обгединение $\bigcup \mathscr{F}$ является $(d-k)$-выпуклым, то семейство $\mathscr{F}$ имеет $k$-трансверсаль. 
ОПредЕЛЕНиЕ 59. Пусть даны два множества $X, Y \subseteq \mathbb{R}^{d}$. Уклонением $X$ от $Y$ называется величина

$$
\delta(X, Y)=\sup _{x \in X} \operatorname{dist}(x, Y) .
$$

Теорема 96 (Карасёв, 2008). Если в $\mathbb{R}^{d}$ дано неантиподальное семейство компактов $\mathscr{F},|\mathscr{F}|=d+1$, то найдется такая $k$-плоскость $M$, что уклонения всех множеств семейства $\mathscr{F}$ от $M$ одинаковы.

Заметим, что в теоремах 95 и 96 расстояние можно брать в любой норме с гладким единичным шаром. Также в работе [100] получены следующие теоремы типа Борсука-Улама-Люстерника-Шнирельмана.

ОПРЕдЕлЕниЕ 60. Для единичной сферы $S^{d-1} \subset \mathbb{R}^{d}$ назовем $k$-nодсферой всякое пересечение $k$-мерного линейного подпространства $L \subseteq \mathbb{R}^{d}$ с $S^{d-1}$.

ОПРЕДЕЛЕНИЕ 61. Для единичной сферы $S^{d-1} \subset \mathbb{R}^{d}$ назовем $k$-nолусферой половину некоторой $k$-подсферы.

Tеорема 97 (Карасёв, 2008). Пусть на сфере $S^{d-1}$ даны d открытых подмножеств $V_{1}, \ldots, V_{d}$, причем всякое $V_{i}$ пересекает всякую $k$-подсферу. Тогда найдется $k$-полусфера, пересекающая все множества $V_{i}$.

Теорема 98 (Карасёв, 2008). Пусть на сфере $S^{d-1}$ дано $d+1$ открытое подмножество $V_{1}, \ldots, V_{d+1}$, причем всякое $V_{i}$ пересекает всякую $k$-подсферу. Тогда либо найдется $k$-полусфера, пересекающая все множества $V_{i}$, либо для всякого разбиения $\{1,2, \ldots, d+1\}=I_{1} \cap I_{2}$ найдется пара $k$-полусфер $H_{1}$ u $H_{2}$, являющихся половинками одной $k$-подсферь, такая, что $V_{i} \cap H_{1}=\varnothing$ для любого $i \in I_{1} \quad u V_{i} \cap H_{2}=\varnothing$ для любого $i \in I_{2}$.

\section{Список литературы}

[1] L.E. J. Brouwer, "Über Abbildung von Mannigfaltigkeiten", Math. Ann., 71:1 (1911), 97-115.

[2] C. Carathéodory, "Über den Variabilitätsbereich der Fourierschen Konstanten von positiven harmonischen Funktionen", Rend. Circ. Mat. Palermo, 32 (1911), 193-217.

[3] J. Radon, "Mengen konvexer Körper, die einen gemeinsamen Punkt enthalten", Math. Ann., 83:1-2 (1921), 113-115.

[4] E. Helly, "Über Mengen konvexer Körper mit gemeinschaftlichen Punkten", Jahresber. Deutsch. Math.-Verein., 32 (1923), 175-176.

[5] E. Sperner, "Neuer Beweis für die Invarianz der Dimensionszahl und des Gebietes", Abh. Math. Sem. Univ. Hamburg, 6:1 (1928), 265-272.

[6] B. Knaster, C. Kuratowski, S. Mazurkiewicz, "Ein Beweis des Fixpunktsatzes für n-dimensionale Simplexe", Fund. Math., 14 (1929), 132-137.

[7] A. W. Tucker, "Some topological properties of disk and sphere", Proceedings of the First Canadian Mathematical Congress (Montreal, 1945), University of Toronto Press, Toronto, 1946, 285-309.

[8] Л.А. Люстерник, Л.Г. Шнирельман, Топологические методы в вариационных задачах, ГНТИ, М., 1930. 
[9] K. Borsuk, "Drei Sätze über die n-dimensionale euklidische Sphäre", Fund. Math., 20 (1933), 177-190.

[10] A. H. Stone, J. W. Tukey, "Generalized 'sandwich' theorems", Duke Math. J., 9:2 (1942), 356-359.

[11] H. Steinhaus, "Sur la division des ensembles de l'espace par les plans et des ensembles plans par les cercles", Fund. Math., 33 (1945), 245-263.

[12] J. Kahn, G. Kalai, "A counterexample to Borsuk's conjecture", Bull. Amer. Math. Soc. (N.S.), 29:1 (1993), 60-62; arXiv: math/9307229, 1993.

[13] B. H. Neumann, "On an invariant of plane regions and mass distributions", J. London Math. Soc., 20:4 (1945), 226-237.

[14] R. Rado, "A theorem on general measure", J. London Math. Soc., 21:4 (1946), 291-300.

[15] Л.Г. Шнирельман, “О некоторых геометрических свойствах замкнутых кривых", УМH, 1944, № 10, 34-44.

[16] S. Kakutani, "A proof that there exists a circumscribing cube around any bounded closed convex set in $\mathbb{R}^{3 "}$, Ann. of Math. (2), 43:4 (1942), 739-741.

[17] R. E. R. van Kampen, "Komplexe in euklidischen Räumen", Abh. Math. Sem. Univ. Hamburg, 9 (1932), 72-78.

[18] A. Flores, "Über $n$-dimensionale Komplexe, die im $R_{2 n+1}$ absolut selbstverschlungen sind", Ergeb. Math. Kolloq., 6 (1935), 4-7.

[19] М. А. Красносельский, "О специальных покрытиях конечномерной сферы”, Докл. АН CCCP, 103 (1955), 961-964.

[20] Chung-Tao Yang, "Continuous functions from spheres to Euclidean spaces", Ann. of Math. (2), 62:2 (1955), 284-292.

[21] Chung-Tao Yang, "On theorems of Borsuk-Ulam, Kakutani-Yamabe-Yujobô and Dyson. II", Ann. of Math. (2), 62:2 (1955), 271-283.

[22] А. С. Шварц, "Род расслоённого пространства", Тр. МMO, 10 (1961), 217-272; 11 (1962), 99-126; англ. пер.: A. S. Schwarz, "The genus of a fibre space", Amer. Math. Soc. Transl., 55 (1966), 49-140.

[23] П. Коннер, Э. Флойд, Гладкие периодические отображения, Мир, М., 1969; пер. с англ.: P. E. Conner, E. E. Floyd, Differentiable periodic maps, Ergeb. Math. Grenzgeb., N.F., 33, Academic Press, New York; Springer-Verlag, Berlin-Göttingen-Heidelberg, 1964.

[24] А. Ю. Воловиков, "К топологическому обобщению теоремы Тверберга", Матем. заметки, 59:3 (1996), 454-456; англ. пер.: A. Yu. Volovikov, "On a topological generalization of the Tverberg theorem", Math. Notes, 59:3 (1996), 324-326.

[25] У И Сян, Когомологическая теория топологических групп преобразований, Мир, М., 1979; англ. пер.: Wu-yi Hsiang, Cohomology theory of topological transformation groups, Ergeb. Math. Grenzgeb., 85, Springer-Verlag, New York-Heidelberg, 1975.

[26] А. Ю. Воловиков, “Теорема типа Буржена-Янга для $\mathbb{Z}_{p}^{n}$-действия", Матем. сб., 183:7 (1992), 115-144; англ. пер.: A. Yu. Volovikov, "A theorem of Bourgin-Yang type for $\mathbb{Z}_{p}^{n}$-action", Russian Acad. Sci. Sb. Math., 76:2 (1993), 361-387.

[27] А. Ю. Воловиков, Е.В. Щепин, “Антиподы и вложения", Матем. сб., 196:1 (2005), 3-32; англ. пер.: А. Yu. Volovikov, E. V. Shchepin, "Antipodes and embeddings", Sb. Math., 196:1 (2005), 1-28.

[28] А. Ю. Воловиков, “Точки совпадения отображений $\mathbb{Z}_{p}^{k}$-пространств в $C W$-комплексы", УМН, 57:1 (2002), 153-154; англ. пер.: А. Yu. Volovikov, "Coincidence points of functions from $\mathbb{Z}_{p}^{k}$-spaces to $C W$-complexes", Russian Math. Surveys, $\mathbf{5 7 : 1}$ (2002), 170-172.

[29] T. Bartsch, Topological methods for variational problems with symmetries, Lecture Notes in Math., 1560, Springer-Verlag, Berlin, 1993. 
[30] G. Carlsson, "Equivariant stable homotopy and Segal's Burnside ring conjecture", Ann. of Math. (2), 120:2 (1984), 189-224.

[31] E. Fadell, S. Husseini, "An ideal-valued cohomological index theory with applications to Borsuk-Ulam and Bourgin-Yang theorems", Ergodic Theory Dynam. Systems, 8 (1988), 73-85.

[32] J. McCleary, A user's guide to spectral sequences, Cambridge Stud. Adv. Math., 58, Cambridge Univ. Press, Cambridge, 2001.

[33] R. N. Karasev, "Periodic billiard trajectories in smooth convex bodies", Geom. Funct. Anal. (to appear).

[34] K. Браун, Когомологии групn, Наука, M., 1987; пер. с англ.: K. S. Brown, Cohomology of groups, Grad. Texts in Math., 87, Springer, New York-Berlin, 1982.

[35] А. Ю. Воловиков, “Точки совпадения отображений $\mathbb{Z}_{p}^{n}$-пространств”, Изв. РАН. Сер. матем., 69:5 (2005), 53-106; англ. пер.: A. Yu. Volovikov, "Coincidence points of maps of $\mathbb{Z}_{p}^{n}$-spaces", Izv. Math., 69:5 (2005), 913-962.

[36] B. Knaster, "Problem 4", Colloq. Math., 30 (1947), 30-31.

[37] B. S. Kashin, S. J. Szarek, "The Knaster problem and the geometry of high-dimensional cubes", C. R. Math. Acad. Sci. Paris, 336:11 (2003), 931-936.

[38] A. Hinrichs, Ch. Richter, "The Knaster problem: more counterexamples", Israel J. Math., 145:1 (2005), 311-324.

[39] H. Yamabe, Z. Yujobô, "On the continuous function defined on a sphere", Osaka Math. J., 2:1 (1950), 19-22.

[40] E. E. Floyd, "Real-valued mappings of spheres", Proc. Amer. Math. Soc., 6:6 (1955), 957-959.

[41] В. В. Макеев, "Задача Кнастера и почти сферические сечения", Матем. сб., 180:3 (1989), 424-431; англ. пер.: V. V. Makeev, "The Knaster problem and almost spherical sections", Math. USSR-Sb., 66:2 (1990), 431-438.

[42] А. Ю. Воловиков, "Об одном свойстве функций на сфере", Матем. заметки, 70:5 (2001), 679-690; англ. пер.: А. Yu. Volovikov, "On a property of functions on the sphere", Math. Notes, 70:5-6 (2001), 616-627.

[43] H. Hopf, "Eine Verallgemeinerung bekannter Abbildungs- und Überdeckungssätze", Port. Math., 4 (1944), 129-139.

[44] В. В. Макеев, "О некоторых свойствах непрерывных отображений сфер и задачах комбинаторной геометрии", Геометрические вопросы теории функиий и множеств, Калинин. гос. ун-т, Калинин, 1986, 75-85.

[45] И.К. Бабенко, С. А. Богатый, "К отображению сферы в евклидово пространство", Матем. заметки, 46:3 (1989), 3-8; англ. пер.: I. K. Babenko, S. A. Bogatyi, "Mapping a sphere into Euclidean space", Math. Notes, 46:3 (1989), 683-686.

[46] W. Chen, "Counterexamples to Knaster's conjecture", Topology, 37:2 (1998), 401-405.

[47] А. Ю. Воловиков, "Об индексе $G$-пространств”, Матем. сб., 191:9 (2000), 3-22; англ. пер.: А. Yu. Volovikov, "On the index of $G$-spaces", Sb. Math., 191:9 (2000), 1259-1277.

[48] M. Kneser, "Aufgabe 360", Jahresbericht der Deutschen Mathematiker-Vereinigung, $\mathbf{5 8 : 2}(1955), 27$.

[49] L. Lovász, "Kneser's conjecture, chromatic number and homotopy", J. Combin. Theory Ser. A, 25:3 (1978), 319-324.

[50] I. Bárány, "A short proof of Kneser's conjecture", J. Combin. Theory Ser. A, 25:3 (1978), 325-326.

[51] В. Л. Дольников, "О трансверсалях семейств выпуклых множеств", Исследования по теории функций многих вещественных переменных, Ярославль, 1981, $30-36$. 
[52] N. Alon, P. Frankl, L. Lovász, "The chromatic number of Kneser hypergraphs", Trans. Amer. Math. Soc., 298:1 (1986), 359-370.

[53] D. Kozlov, Combinatorial algeraic topology, Algorithms Comput. Math., 21, Springer, Berlin, 2008.

[54] I. Kříž, "Equivariant cohomology and lower bounds for chromatic numbers", Trans. Amer. Math. Soc., 333:2 (1992), 567-577.

[55] I. Kř́žz, "A correction to 'Equivariant cohomology and lower bounds for chromatic numbers"', Trans. Amer. Math. Soc., 352:4 (2000), 1951-1952.

[56] G. M. Ziegler, "Generalized Kneser coloring theorems with combinatorial proofs", Invent. Math., 147:3 (2002), 671-691.

[57] А. Ю. Воловиков, "Род $G$-пространств и нижняя топологическая оценка хроматического числа гиперграфа", Фундамент. и прикл. матем., 11:4 (2005), 33-48; англ. пер.: А. Yu. Volovikov, "The genus of $G$-spaces and topological lower bounds for chromatic numbers of hypergraphs", J. Math. Sci. (N. Y.), 144:5 (2007), 4387-4397.

[58] H. Tverberg, "A generalization of Radon's theorem", J. London Math. Soc., 41:1 (1966), 123-128.

[59] I. Bárány, "A generalization of Carathéodory's theorem", Discrete Math., 40:2-4 (1982), 141-152.

[60] I. Bárány, D. G. Larman, "A colored version of Tverberg's theorem", J. London Math. Soc., 45:2 (1992), 314-320.

[61] B. Eaves, "Homotopies for computation of fixed points", Math. Program., 3:1 (1972), $1-22$.

[62] R. B. Kellogg, T. Li, J. Yorke, "Constructive proof of the Brouwer fixed-point theorem and computational results", SIAM J. Numer. Anal., 13:4 (1976), 473-483.

[63] I. Bárány, S. B. Shlosman, S. Szücs, "On a topological generalization of a theorem of Tverberg", J. London Math. Soc. (2), 23:1 (1981), 158-164.

[64] А. Ю. Воловиков, "К теореме ван Кампена-Флореса", Матем. заметки, 59:5 (1996), 663-670; англ. пер.: A. Yu. Volovikov, "On the van Kampen-Flores theorem", Math. Notes, 59:5 (1996), 477-481.

[65] K.S. Sarkaria, "A generalized van Kampen-Flores theorem", Proc. Amer. Math. Soc., 111:2 (1991), 559-565.

[66] R. T. Živaljević, S. T. Vrećica, "The colored Tverberg's problem and complexes of injective functions", J. Combin. Theory Ser. A, 61:2 (1992), 309-318.

[67] R. T. Živaljević, S. T. Vrećica, "New cases of the colored Tverberg theorem", Jerusalem combinatorics (1993), Contemp. Math., 178, eds. H. Barcelo, G. Kalai, Amer. Math. Soc., Providence, RI, 1994, 325-334.

[68] Р.Н. Карасёв, “Двойственные теоремы о центральной точке и их обобщения”, Матем. сб., 199:10 (2008), 41-62.

[69] H. Guggenheimer, "Finite sets on curves and surfaces", Israel J. Math., 3:2 (1965), 104-112.

[70] R. P. Jerrard, "Inscribed squares in plane curves", Trans. Amer. Math. Soc., 98:2 (1961), 234-241.

[71] I. Pak, The discrete square peg problem, arXiv: 0804.0657v1.

[72] М. Л. Громов, "О симплексах, вписанных в гиперповерхности", Матем. заметкu, 5:1 (1969), 81-89; англ. пер.: M. L. Gromov, "On simplexes inscribed in a hypersurface", Math. Notes, 5 (1969), 52-56.

[73] В. В. Макеев, Универсально вписанные и описанные многогранники, Дисс. ... докт. физ.-матем. наук, Санкт-Петербургский государственный университет, Санкт-Петербург, 2003. 
[74] В. В. Макеев, "Аффинно-вписанные и аффинно-описанные многоугольники и многогранники", Записки науч. сем. ПОМИ, 231 (1995), 286-298; англ. пер.: V.V. Makeev, "Affine-inscribed and affine-circumscribed polygons and polytopes", J. Math. Sci. (New York), 91:6 (1998), 3518-3525.

[75] J. F. Pál, "Über ein elementares Variationprobleme", Bull. de l'Acad. de Dan., 3:2 (1920), 35 pp.

[76] H. G. Eggleston, Convexity, Cambridge Tracts in Math. and Math. Phys., 47, Cambridge Univ. Press, New York, 1958.

[77] D. Gale, "On inscribing $n$-dimensional sets in a regular $n$-simplex", Proc. Amer. Math. Soc., 4:2 (1953), 222-225.

[78] В. В. Макеев, "Об аффинных образах ромбододекаэдра, описанных вокруг трехмерного выпуклого тела”, Записки науч. сем. ПОМИ, 246 (1997), 191-195; англ. пер.: V.V. Makeev, "Affine images of the rhombo-dodecahedron that are circumscribed about a three-dimensional convex body", J. Math. Sci. (New York), 100:3 (2000), 2307-2309.

[79] G. Kuperberg, "Circumscribing constant-width bodies with polytopes", New York J. Math., 5 (1999), 91-100.

[80] Р.Н.Карасёв, "Вписывание правильного кроссполитопа", Матем. заметки (в печати).

[81] G. D. Birkhoff, Dynamical systems, Amer. Math. Soc. Colloq. Publ., 9, Amer. Math. Soc., New York, 1927.

[82] N. H. Kuiper, "Double normals of convex bodies", Israel J. Math., 2:2 (1964), 71-80.

[83] И.К. Бабенко, "Периодические траектории трехмерных бильярдов Биркгофа", Матем. сб., 181:9 (1990), 1155-1169; англ. пер.: I. K. Babenko, "Periodic trajectories in three-dimensional Birkhoff billiards", Math. USSR-Sb., 71:1 (1992), $1-13$.

[84] M. Farber, S. Tabachnikov, "Topology of cyclic configuration spaces and periodic trajectories of multi-dimensional billiards", Topology, 41:3 (2002), 553-589.

[85] M. Farber, "Topology of billiard problems. II", Duke Math. J., 115:3 (2002), 587-621.

[86] B. Grünbaum, "Partitions of mass-distributions and of convex bodies by hyperplanes", Pacific J. Math., 10 (1960), 1257-1261.

[87] E. A. Ramos, "Equipartition of mass distributions by hyperplanes", Discrete Comput. Geom., 15:2 (1996), 147-167.

[88] H. Hadwiger, "Simultane Vierteilung zweier Körper", Arch. Math. (Basel), 17:3 (1966), 274-278.

[89] D. Avis, "Non-partitionable point sets", Inform. Process. Lett., 19:3 (1984), 125-129.

[90] P. Mani-Levitska, S. Vrećica, R. Živaljević, "Topology and combinatorics of partitions of masses by hyperplanes", Adv. Math., 207:1 (2006), 266-296.

[91] В. В. Макеев, "Вписанные и описанные многогранники выпуклого тела", Maтем. заметки, 55:4 (1994), 128-130; англ. пер.: V. V. Makeev, "Inscribed and circumscribed polyhedra of a convex body", Math. Notes, 55:4 (1994), 423-425.

[92] V. V. Makeev, "Applications of topology to some problems in combinatorial geometry", Amer. Math. Soc. Transl. Ser. 2, 174, Amer. Math. Soc., Providence, RI, 1996, 223-228.

[93] S. T. Vrećica, R. T. Živaljević, "The ham sandwich theorem revisited", Israel J. Math., 78:1 (1992), 21-32.

[94] S. T. Vrećica, R. T. Živaljević, "Conical equipartitions of mass distributions", Discrete Comput. Geom., 25:3 (2001), 335-350.

[95] N. Alon, "Splitting necklaces", Adv. Math., 63:3 (1987), 247-253.

[96] A. Vućić, R. T. Živaljević, "Note on a conjecture of Sierksma", Discrete Comput. Geom., 9:4 (1993), 339-349. 
[97] M. de Longueville, R. T. Živaljević, "Splitting multidimensional necklaces", Adv. Math., 218:3 (2008), 926-939.

[98] В. В. Макеев, "Шестидольные разбиения трехмерного пространства", Вестник ЛГУ, 2 (1988), 31-34; англ. пер.: V.V. Makeev, "Six-partite partitions of a three-dimensional space", Vestnik Leningrad Univ. Math., 21:2 (1988), 40-45.

[99] I. Bárány, A. Hubard, J. Jerónimo, "Slicing convex sets and measures by a hyperplane", Discrete Comput. Geom., 39:1-3 (2008), 67-75.

[100] Р.Н. Карасёв, “Теоремы типа Борсука-Улама для плоскостей и плоские трансверсали семейств выпуклых компактов", Матем. сб. (в печати).

[101] R. N. Karasev, "Partitions of a polytope and mappings of a point set to facets", Discrete Comput. Geom., 34:1 (2005), 25-45.

[102] J. Eckhoff, "Helly, Radon, and Carathéodory type theorems", Handbook of convex geometry, Vol. A, B, North-Holland, Amsterdam, 1993, 389-448.

[103] J. Leray, "Sur la forme des espaces topologique et sur les points fixes des représentations", J. Math. Pures Appl. (9), 24:9 (1945), 95-167.

[104] G. Wegner, "d-collapsing and nerves of families of convex sets", Arch. Math. (Basel), 26:1 (1975), 317-321.

[105] G. Wegner, Eigenschaften der Nerven homologisch-einfacher Familien im $\mathbb{R}^{n}, \mathrm{Ph}$. D. Thesis, University of Göttingen, 1967.

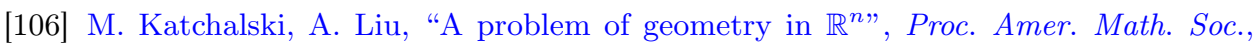
75:2 (1979), 284-288.

[107] H. Hadwiger, H. Debrunner, "Über eine Variante zum Hellyschen Satz", Arch. Math., 8:4 (1957), 309-313.

[108] N. Alon, D. J. Kleitman, "Piercing convex sets and the Hadwiger-Debrunner (p,q)-problem", Adv. Math., 96:1 (1992), 103-112.

[109] N. Alon, G. Kalai, J. Matoušek, R. Meshulam, "Transversal numbers for hypergraphs arising in geometry", Adv. in Appl. Math., 29:1 (2002), 79-101.

[110] G. Kalai, R. Meshulam, "A topological colorful Helly theorem", Adv. Math., 191:2 (2005), 305-311.

[111] R. B. Bapat, "A constructive proof of a permutation-based generalization of Sperner's lemma", Math. Program., 44:1-3 (1989), 113-120.

[112] Р. Н. Карасёв, "Раскрашенная версия леммы Кнастера-Куратовского-Мазуркевича", Моделирование и анализ информаиионных систем, 13:2 (2006), 66-70.

[113] G. Tardos, "Transversals of 2-intervals, a topological approach", Combinatorica, 15:1 (1995), 123-134.

[114] G. Tardos, "Trasversals of $d$-intervals - comparing three approaches", European Congress of Mathematics, Vol. II (Budapest, 1996), Progr. Math., 169, Birkhäuser, Basel, 1998, 234-243.

[115] H. Hadwiger, "Über Eibereiche mit gemeinsamer Treffgeraden", Port. Math., 16 (1957), 23-29.

[116] M. Katchalski, T. Lewis, "Cutting families of convex sets", Proc. Amer. Math. Soc., 79:3 (1980), 457-461.

[117] H. Tverberg, "Proof of Grünbaum's conjecture on common transversals for translates", Discrete Comput. Geom., 4:3 (1989), 191-203.

[118] A. Holmsen, M. Katchalski, T. Lewis, "A Helly-type theorem for line transversals to disjoint unit balls", Discrete Comput. Geom., 29:4 (2003), 595-602.

[119] O. Cheong, X. Goaoc, A. Holmsen, S. Petitjean, "Helly-type theorems for line transversals to disjoint unit balls", Discrete Comput. Geom., 39:1-3 (2008), 194-212.

[120] A. Holmsen, J. Matoušek, "No Helly theorem for stabbing translates by lines in $\mathbb{R}^{3}$ ", Discrete Comput. Geom., 31:3 (2004), 405-410. 
[121] B. Aronov, J. E. Goodman, R. Pollack, R. Wenger, "On the Helly number for hyperplane transversals to unit balls", Discrete Comput. Geom., 24:2-3 (2000), 171-176.

[122] J. E. Goodman, R. Pollack, "Hadwiger's transversal theorem in higher dimensions", J. Amer. Math. Soc., 1:2 (1988), 301-309.

[123] A. Holmsen, "The Katchalski-Lewis transversal problem in $\mathbb{R}^{n ", ~ D i s c r e t e ~ C o m p u t . ~}$ Geom., 37:3 (2007), 341-349.

[124] A. Horn, "Some generalization of Helly's theorem on convex sets", Bull. Amer. Math. Soc., 55 (1949), 923-929.

[125] V. Klee, "On certain intersection properties of convex sets", Canad. J. Math., 3 (1951), 272-275.

[126] В.Л. Дольников, "Обобщенные трансверсали семейств множеств в $\mathbb{R}^{n}$ и связи между теоремами Хелли и Борсука”, Докл. АН CCCP, 297:4 (1987), 777-780; англ. пер.: V. L. Dol'nikov, "Generalized transversals of families of sets in $\mathbb{R}^{n}$ and the connections between the Helly theorem and the Borsuk theorem", Soviet Math. Dokl., 36:3 (1988), 519-522.

[127] R. T. Živaljević, "The Tverberg-Vrećica problem and the combinatorial geometry on vector bundles", Israel J. Math., 111:1 (1999), 53-76.

[128] R. N. Karasev, "Tverberg's transversal conjecture and analogues of nonembeddability theorems for transversals", Discrete Comput. Geom., 38:3 (2007), 513-525.

[129] H. L. Hiller, "On the cohomology of real Grassmanians", Trans. Amer. Math. Soc., 257:2 (1980), 521-533.

[130] H. L. Hiller, "On the height of the first Stiefel-Whitney class", Proc. Amer. Math. Soc., 79:3 (1980), 495-498.

[131] М.Л. Громов, “Об одной геометрической гипотезе Банаха”, Изв. АН CCCP. Cер. матем., 31:5 (1967), 1105-1114; англ. пер.: M. L. Gromov, "A geometrical conjecture of Banach", Math. USSR-Izv., 1:5 (1967), 1055-1064.

[132] E. Makai, S. Vrećica, R. Živaljević, "Plane sections of convex bodies of maximal volume", Discrete Comput. Geom., 25:1 (2001), 33-49.

[133] R. T. Živaljević, S. T. Vrećica, "An extension of the ham sandwich theorem", Bull. London Math. Soc., 22:2 (1990), 183-186.

[134] В. Л. Дольников, "О разбиении системы мер подпространством многочленов", Конструирование алгоритмов и решение задач математической физики, Доклады 8-го Всесоюзного семинара "Теоретические основы и конструирование численных алгоритмов решения задач математической физики" (Красновидово, 7-11 октября 1990), ИПМ им. М. В. Келдыша, М., 1991, 80-85.

[135] В. Л. Дольников, "Об одном обобщении теоремы о бутерброде", Матем. заметки, 52:2 (1992), 27-37; англ. пер.: V. L. Dol'nikov, "A generalization of the ham sandwich theorem", Math. Notes, 52:2 (1992), 771-779.

[136] H. Tverberg, S. Vrećica, "On generalizations of Radon's theorem and the ham sandwich theorem", European J. Combin., 14:3 (1993), 259-264.

[137] S. T. Vrećica, "Tverberg's conjecture", Discrete Comput. Geom., 29:4 (2003), 505-510.

P. H. Kapacëв (R. N. Karasev)

Московский физико-технический институт

(государственный университет)

E-mail: r_n_karasev@mail.ru
Поступила в редакцию

07.10 .2008 